\title{
Sample Size Planning for the Squared Multiple Correlation Coefficient: Accuracy in Parameter Estimation via Narrow Confidence Intervals
}

\author{
Ken Kelley \\ Department of Management \\ University of Notre Dame
}

\begin{abstract}
Methods of sample size planning are developed from the accuracy in parameter approach in the multiple regression context in order to obtain a sufficiently narrow confidence interval for the population squared multiple correlation coefficient when regressors are random. Approximate and exact methods are developed that provide necessary sample size so that the expected width of the confidence interval will be sufficiently narrow. Modifications of these methods are then developed so that necessary sample size will lead to sufficiently narrow confidence intervals with no less than some desired degree of assurance. Computer routines have been developed and are included within the MBESS R package so that the methods discussed in the article can be implemented. The methods and computer routines are demonstrated using an empirical example linking innovation in the health services industry with previous innovation, personality factors, and group climate characteristics.
\end{abstract}

In the behavioral, educational, managerial, and social (BEMS) sciences, one of the most commonly used statistical methods is multiple regression. When designing studies that will use multiple regression, sample size planning is often considered by researchers before the start of a study in order to ensure there

Correspondence concerning this article should be addressed to Ken Kelley, Department of Management, Mendoza College of Business, University of Notre Dame, Notre Dame, IN 46556. E-mail: KKelley@ND.Edu 
is adequate statistical power to reject the null hypothesis that the population squared multiple correlation coefficient (denoted with an uppercase rho squared, $\mathrm{P}^{2}$ ) equals zero. Planning sample size from this perspective is well known in the sample size planning literature (e.g., Cohen, 1988; Dunlap, Xin, \& Myers, 2004; Gatsonis \& Sampson, 1989; Green, 1991; Mendoza \& Stafford, 2001). However, with the exception of Algina and Olejnik (2000), sample size planning when interest concerns obtaining accurate estimates of $\mathrm{P}^{2}$ has largely been ignored. With the emphasis that is now being placed on confidence intervals for effect sizes in the literature, and with the desire to avoid "embarrassingly large" confidence intervals (Cohen, 1994, p. 1002), planning sample sizes so that one can achieve narrow confidence intervals continues to increase in importance. Planning sample size when one is interested in $\mathrm{P}^{2}$ can thus proceed in (at least) two fundamentally different ways: (a) one that plans an appropriate sample size based on some desired degree of statistical power for rejecting the null hypothesis that $\mathrm{P}^{2}=0$ (or some other specified value) and (b) one that plans an appropriate sample size in order for the confidence interval for $\mathrm{P}^{2}$ to be sufficiently narrow.

The purpose of this article is to provide an alternative to the power analytic approach to sample size planning for the squared multiple correlation coefficient in the accuracy in parameter estimation (AIPE) context (Kelley, 2007c; Kelley \& Maxwell, 2003, 2008; Kelley, Maxwell, \& Rausch, 2003; Kelley \& Rausch, 2006; Maxwell, Kelley, \& Rausch, 2008). The general idea of the AIPE approach to sample size planning is to obtain a confidence interval that is sufficiently narrow at some specified level of coverage and thus avoid wide confidence intervals, which illustrate the uncertainty with which the parameter has been estimated. The theoretical differences between the AIPE and power analytic approaches to sample size planning and the implications for the cumulative knowledge of an area are delineated in Maxwell et al. (2008). ${ }^{1}$

As recommended by Wilkinson and the American Psychological Association (APA) Task Force on Statistical Inference (1999), researchers should "always present effect sizes for primary outcomes" (p. 599). Similarly, the American Educational Research Association recently adopted reporting standards that state, "An index of the quantitative relation between variables" (i.e., an effect size) and "an indication of the uncertainty of that index of effect" (i.e., a confidence interval) should be included when reporting statistical results (2006, p. 10). Although not true for effect sizes in general, BEMS researchers have tended to report the estimated squared multiple correlation coefficient. However, Wilkinson and the APA Task Force go on to recommended that "interval estimates should

${ }^{1}$ The use of the term "accuracy" in this context is the same as that used by Neyman (1937) in his seminal work on the theory of confidence interval construction: "The accuracy of estimation corresponding to a fixed value of $1-\alpha$ may be measured by the length of the confidence interval" (p. 358; notation changed to reflect current usage). 
be given for any effect sizes involving principal outcomes" (1999, p. 599), something that has not historically been done. This is not a problem unique to BEMS research: it seems confidence intervals for $\mathrm{P}^{2}$ have not historically been reported in any domain of research. Nevertheless, with increased emphasis on confidence intervals for effect sizes, there is almost certainly going to be an increase in the use of confidence intervals for $\mathrm{P}^{2}$. Indeed, confidence intervals for effect sizes may well be the future of quantitative research in the BEMS sciences (Thompson, 2002).

One problem waiting to manifest itself when researchers routinely begin to report confidence intervals for $\mathrm{P}^{2}$ is that, even with sufficient statistical power, the widths of the confidence interval for $\mathrm{P}^{2}$ might be large, illustrating the uncertainty with which information is known about $\mathrm{P}^{2}$. This article fills a void in the multiple regression and sample size planning literatures by developing methods so that sample size can be planned when there is an interest in achieving a narrow confidence interval for $\mathrm{P}^{2}$. The first method developed yields necessary sample size so that the expected width of the obtained confidence interval for $\mathrm{P}^{2}$ is sufficiently narrow. For example, a researcher may plan sample size so that the expected width of the confidence interval for $\mathrm{P}^{2}$ is .10 . Because the confidence interval width is itself a random variable based in part on $R^{2}$, the usual (although biased) estimate of $\mathrm{P}^{2}$, the observed width is not guaranteed to be sufficiently narrow even though its expected width is sufficiently narrow. A modified approach yields the necessary sample size so that the confidence interval for $\mathrm{P}^{2}$ will be sufficiently narrow with some desired degree of assurance, where the assurance is a probabilistic statement. For example, a researcher may plan sample size so that the confidence interval for $\mathrm{P}^{2}$ is no wider than .10 with 99\% assurance.

Multiple regression is used with fixed and/or random regressors. Distributional characteristics of regression coefficients are different for fixed and random regressors due to the increased randomness in the model when regressors are random because of the sample-to-sample variability (e.g., Gatsonis \& Sampson, 1989; Rencher, 2000; Sampson, 1974). ${ }^{2}$ The sample size procedures developed here are for the case where regressors are random, which is how multiple regression is generally used in the BEMS sciences. Confidence intervals for $\mathrm{P}^{2}$ have not often been considered in applied work (but see Algina \& Olejnik, 2000; Ding, 1996; Kelley \& Maxwell, 2008; Lee, 1971; Mendoza \& Stafford, 2001; Smithson, 2001; and Steiger \& Fouladi, 1992, for discussions and procedures of confidence interval formation for $\mathrm{P}^{2}$ ). However, with the strong

\footnotetext{
${ }^{2}$ The term "regressors" is used as a generic term to denote the $K X$ variables. In other contexts the regressors are termed independent, explanatory, predictor, or concomitant variables. The term "criterion" is used as a generic term for the variable that is modeled as a function of the $K$ regressors. In other contexts, the criterion variable is termed dependent, outcome, or predicted variable.
} 
encouragement from methodologists and important professional associations, as well as the development and recognition of software to implement confidence intervals for $\mathrm{P}^{2}$, there is likely to be an increase in reporting confidence intervals for $\mathrm{P}^{2}$.

The article begins with a discussion of confidence interval formation for $\mathrm{P}^{2}$ and idiosyncratic estimation issues that become important when the sample size procedures are developed. Development of sample size planning for AIPE in the context of $\mathrm{P}^{2}$ is then given. Results of a Monte Carlo simulation study are summarized that illustrate the effectiveness of the proposed procedures. The procedures used and methods developed in this article can be easily implemented in the program $\mathrm{R}$ (R Development Core Team, 2008) with the MBESS package (Kelley, 2007a, 2007b, 2008; Kelley, Lai, \& Wu, 2008). A demonstration of the methods developed is given using an empirical example, where the methods are implemented with MBESS in the context of innovation in the health services industry linking innovation with previous innovation, personality factors, and group climate characteristics (Bunce \& West, 1995). Because the value of $\mathrm{P}^{2}$ is of interest in many applications of multiple regression, not literally the dichotomous reject or fail to reject decision of a null hypothesis significance test, it is hoped that researchers will consider the AIPE approach instead of or in addition to the power analytic approach when planning a study where multiple regression will be used to analyze data.

\section{ESTIMATION AND CONFIDENCE INTERVAL FORMATION FOR $\mathrm{P}^{2}$}

The common estimate of $\mathrm{P}^{2}, R^{2}$, is positively biased and thus tends to overestimate the degree of linear relationship between the $K$ regressor variables and the criterion variable in the population, especially when sample size, $N$, is very small. The expected value of $R^{2}$ given $\mathrm{P}^{2}, N$, and $K$ is given as

$$
\mathrm{E}\left[R^{2} \mid\left(\mathrm{P}^{2}, N, p\right)\right]=1-\frac{N-K-1}{N-1}\left(1-\mathrm{P}^{2}\right) \mathrm{H}\left[1 ; 1 ; \frac{N+1}{2} ; \mathrm{P}^{2}\right]
$$

for multivariate normal data, where $\mathrm{H}$ is the hypergeometric function (Johnson, Kotz, \& Balakrishnan, 1995, p. 621; Stuart, Ord, \& Arnold, 1999, section 28.32). For notational ease, $\mathrm{E}\left[R^{2} \mid\left(\mathrm{P}^{2}, N, p\right)\right]$ will be written as $\mathrm{E}\left[R^{2}\right]$.

As discussed by Steiger (2004), "confidence intervals for the squared multiple correlation are very informative yet are not discussed in standard texts, because a single simple formula for the direct calculation of such an interval cannot be obtained in a manner that is analogous to the way one obtains a confidence interval for the population mean" (p. 167). The difficulties when forming a 
confidence interval for $\mathrm{P}^{2}$ arise for several reasons. First, there is no closed form solution for confidence intervals for $\mathrm{P}^{2}$. The lack of closed form solutions requires iterative algorithms for forming the confidence intervals. Second, there is a nontrivial difference in the distribution of $R^{2}$ when regressors are fixed compared with when they are random when $\mathrm{P}^{2}>0$. Fixed regressors are specified a priori as part of the design of the study. These values are "fixed" in the sense that over theoretical replications of the study, the values of the regressors would not change. This is contrasted to the situation of random regressors, where the regressors are a function of the particular sample. These values are "random" in the sense that over theoretical replications of the study, each sample would yield a different set of regressors. This article focuses only on the case of random regressors, as applications of multiple regression in the BEMS sciences tend to be random. Although confidence intervals for fixed regressor variables are fairly straightforward given the appropriate noncentral $F$-distribution routines, confidence interval formation is much more difficult when regressors are random.

Sampson (1974) and Gatsonis and Sampson (1989) provide a discussion of the differences between the fixed and the random regressor models for multiple regression. Lee (1971) and Ding (1996) provide algorithms for computing various aspects of the distribution of $R^{2}$ for random regressors, which can be used in order to compute confidence intervals. Although the programming of the algorithms underlying the computer programs is not trivial, nearly exact confidence intervals for $\mathrm{P}^{2}$ under the case of random regressors variables can be found with the use of freely available software (e.g., MBESS; Kelley, 2008; MultipleR2; Mendoza \& Stafford, 2001; R2; Steiger \& Fouladi, 1992) and in other programs indirectly with proper programming (e.g., in SAS; Algina \& Olejnik, 2000).

Unlike many effect sizes, there is not a monotonic relation between the width of the confidence interval for $\mathrm{P}^{2}$ and the value of $R^{2}$. An illustration between the relation of $R^{2}$ and the confidence interval width is shown in Figure 1. The values on the abscissa are hypothetical observed values of $R^{2}$ with the $95 \%$ confidence interval width on the ordinate for a sample size of 100 and five regressor variables. The confidence interval width, $w$, is simply the value of the upper limit minus the lower limit,

$$
w=\mathrm{P}_{U}^{2}-\mathrm{P}_{L}^{2}
$$

where $\mathrm{P}_{U}^{2}$ is the upper limit of the confidence interval for $\mathrm{P}^{2}$, and $\mathrm{P}_{L}^{2}$ is the lower limit of the confidence interval for $\mathrm{P}^{2}$ (note that both $\mathrm{P}_{L}^{2}$ and $\mathrm{P}_{U}^{2}$ are random quantities that depend on $R^{2}$ ). Although the exact value of $R^{2}$ for the maximum confidence interval width depends on sample size and the number of regressors, it approaches .333 as sample size increases; although no proof of this is given, 


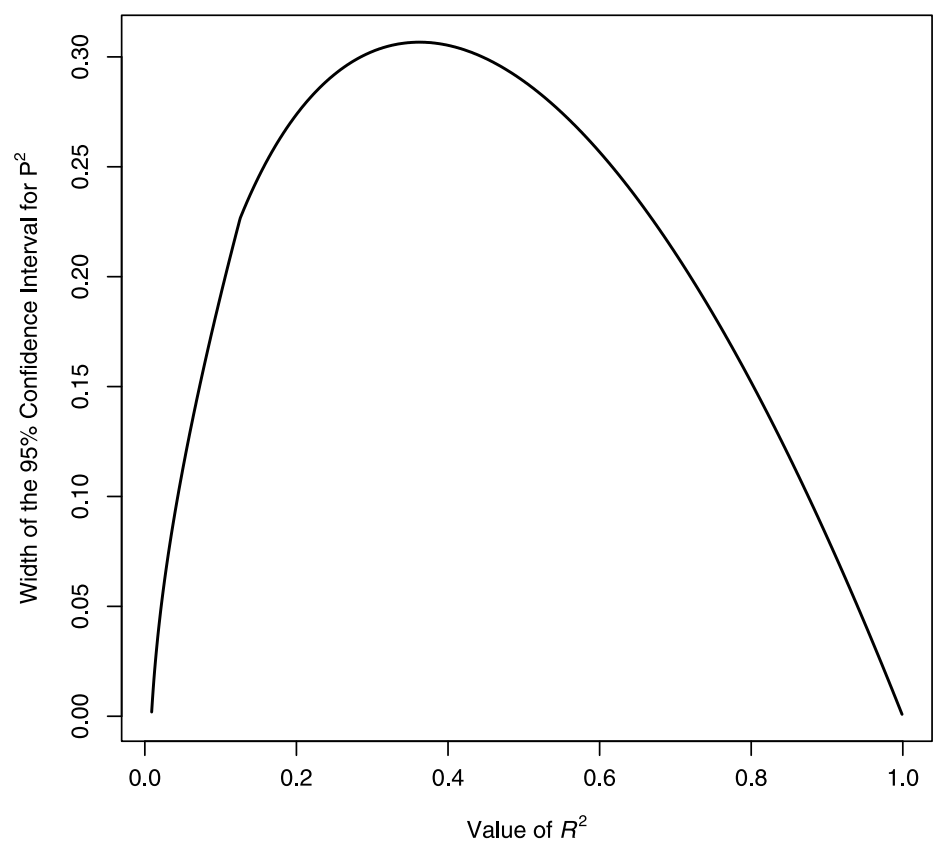

FIGURE 1 Relationship between the confidence interval width for $\mathrm{P}^{2}$ given $N=100$ and $K=5$ for all possible values of $R^{2}$.

the reason is alluded to in Algina and Olejnik (2000), where it can be shown that the approximate variance of $R^{2}$ is maximized at $R^{2}=.333$ (p. 125).

Thus, Figure 1 clearly illustrates that there is a nonmonotonic relationship between confidence interval width and $R^{2}$. Usually confidence interval width is independent of the size of the effect or the confidence interval width increases (decreases) as the absolute value of the of the effect increases (decreases). Notice in Figure 1 that confidence intervals for $\mathrm{P}^{2}$ increase to a peak and then decrease. For very small or very large values of $R^{2}$ the confidence intervals are narrow. For values of $K$ other than five and for sample sizes that are not exceedingly small, combinations of confidence interval width and the size of standard errors tend to be similar to the relationship displayed in Figure 1. The nonmonotonic relationship between $R^{2}$ and $w$ displayed in Figure 1 becomes important in a future section when determining necessary sample size for obtaining a confidence interval no wider than desired with some desired degree of assurance. Given the figure and the fact that the approximate variance of $R^{2}$ is maximized close to .333 , it is no surprise that values of $\mathrm{P}^{2}$ close to .333 will mandate a larger sample size than other values of $\mathrm{P}^{2}$ for some desired confidence interval width, holding everything else constant. 


\section{SAMPLE SIZE FOR NARROW CONFIDENCE INTERVALS FOR THE POPULATION SQUARED MULTIPLE CORRELATION COEFFICIENT}

The idea of obtaining a narrow confidence interval for the lower bound of the population multiple correlation, P, was discussed by Darlington (1990), where a small table of necessary sample size for selected conditions was provided in order for the lower confidence limit to be at least some specified value. Algina and Olejnik (2000) discussed a procedure for sample size planning so that the $R^{2}$ would be within some defined range of $\mathrm{P}^{2}$ with a specified probability. The present work develops methods of sample size planning from the AIPE perspective so that the width of confidence intervals for $\mathrm{P}^{2}$ will be sufficiently narrow. Although similar, the AIPE and Algina \& Olejnik methods are actually fundamentally different ways of planning sample size. The sample size planning methods developed by Algina \& Olejnik have as their goal obtaining an estimate of $\mathrm{P}^{2}$ that is within some defined range of $\mathrm{P}^{2}$, whereas the goal of the AIPE approach is to obtain a sufficiently narrow confidence interval. ${ }^{3}$

The width of the confidence interval for $\mathrm{P}^{2}$ is a function of $R^{2}, N, K$, and $1-\alpha$. By holding constant $\mathrm{P}^{2}, K$, and $1-\alpha$, the expected confidence interval width can be determined for different values of $N$. The expected confidence interval width for $\mathrm{P}^{2}$ is defined as the width of the confidence interval for $\mathrm{E}\left[R^{2}\right]$ given a particular $N, K$, and $1-\alpha$,

$$
\mathrm{E}\left[\mathrm{P}_{U}^{2} \mid \mathrm{E}\left[R^{2}\right]\right]-\mathrm{E}\left[\mathrm{P}_{L}^{2} \mid \mathrm{E}\left[R^{2}\right]\right]=\mathrm{E}\left[\left(\mathrm{P}_{U}^{2}-\mathrm{P}_{L}^{2}\right) \mid \mathrm{E}\left[R^{2}\right]\right]=\mathrm{E}\left[w \mid \mathrm{E}\left[R^{2}\right]\right],
$$

where $\mathrm{E}\left[\left(\mathrm{P}_{U}^{2}-\mathrm{P}_{L}^{2}\right) \mid \mathrm{E}\left[R^{2}\right]\right]$ is found by calculating the width of a confidence interval using $\mathrm{E}\left[R^{2}\right]$ in place of $R^{2}$ from the standard confidence interval procedure. ${ }^{4}$ For notational ease, $\mathrm{E}\left[w \mid \mathrm{E}\left[R^{2}\right]\right]$ is written as $\mathrm{E}[w]$, realizing that the expected width will necessarily depend on $1-\alpha$ and $\mathrm{E}\left[R^{2}\right]$, which itself depends on $N$

\footnotetext{
${ }^{3}$ Tables of sample size comparisons between the AIPE and Algina and Olejnik (2000) methods have developed and are available from Ken Kelley.

${ }^{4}$ In the work of Kelley and Rausch (2006), where the AIPE approach was developed for the standardized mean difference, the population value of the standardized mean difference was used throughout the sample size procedures rather than the expected value of the sample standardized mean difference even though the commonly used estimate is biased. This contrasted to the approach here where the $\mathrm{E}\left[R^{2}\right]$ is used in place of $\mathrm{P}^{2}$. As noted in Kelley \& Rausch (2006, footnote 13), for even relatively small sample sizes the bias in the ordinary estimate of the standardized mean difference is minimal and essentially leads to no differences in planned sample size except in unrealistic situations (see also Hedges \& Olkin, 1985, chap. 5). However, the bias between $R^{2}$ and $\mathrm{P}^{2}$ can be large, relatively speaking, which would lead to differences in necessary sample sizes if the procedure was based on $\mathrm{P}^{2}$ directly, as confidence intervals are based on the positively biased value of $R^{2}$.
} 
and $K$. Because in any particular study $N, K$, and $1-\alpha$ are fixed design factors, the only random variable when forming confidence intervals for $\mathrm{P}^{2}$ is $R^{2}$.

For unbiased estimators, calculation of the expected confidence interval width is simple given the population value because the expected value of the estimator and the population parameter it estimates are equivalent. Due to the positive bias of $R^{2}$ as an estimator of $\mathrm{P}^{2}$, basing the sample size procedure on $\mathrm{P}^{2}$ directly would lead to inappropriate estimates of sample size because the obtained confidence interval is ultimately based on $R^{2}$. Therefore, the expected value of $R^{2}$ is used in the calculation of necessary sample size in order to achieve a confidence interval calculated from the obtained $R^{2}$ that is sufficiently narrow. The algorithms used to obtain appropriate sample size are iterative with regard to sample size. Thus, it is necessary for the expected value of $R^{2}$ to be updated for each iteration of the sample size procedure.

\section{Sample Size so That the Expected Confidence Interval Width is Sufficiently Narrow}

Recall that the sample size methods are developed specifically in the case of random regressor variables. The method begins by determining a lower bound (starting) value for sample size, say $N_{(0)}$, where the quantity subscripted in parentheses represents the iteration of the procedure, so that the minimum sample size where $\mathrm{E}[w]$ is no larger than $\omega$ can be found, where $\omega$ is the desired confidence interval width. Thus, the procedure seeks the minimum necessary sample size so that $\mathrm{E}[w] \leq \omega$. For convenience, $N_{(0)}=2 K+1$.

Given $N_{(0)}, \mathrm{E}\left[w_{(0)}\right]$ is calculated, where $\mathrm{E}\left[w_{(0)}\right]$ is the expected confidence interval width based on $N_{(0)}$. If $\mathrm{E}\left[w_{(0)}\right]>\omega$, sample size is incremented by one and $\mathrm{E}\left[w_{(1)}\right]$ determined. This iterative procedure continues until $\mathrm{E}\left[w_{(i)}\right] \leq \omega$, where the corresponding value of $N_{(i)}$ is set to the necessary sample size with $i$ representing the particular iteration of the procedure. Thus, the $N_{(i)}$ where $\mathrm{E}\left[w_{(i)}\right] \leq \omega$ is set to the necessary sample size. The rationale of this approach is based on the fact that $w$ is a function of $R^{2}$, so finding the necessary sample size based on $\mathrm{E}\left[R^{2}\right]$ that leads to $\mathrm{E}\left[w_{(i)}\right] \leq \omega$ leads to the necessary sample size.

A sample size planning procedure was developed in this section so that the expected confidence interval width for $\mathrm{P}^{2}$ is sufficiently narrow. No formal mathematical proof is known to exist and the procedure may not always give exactly the correct sample size. However, a follow-up procedure developed later exists that updates the (approximate) sample size to the exact value based on an a priori Monte Carlo simulation study. Given the sample size that leads to an expected confidence interval width being sufficiently narrow in the procedure described earlier, provided $\mathrm{P}^{2}$ has been properly specified, the sample size obtained will ensure that the expected confidence interval width is sufficiently 
narrow. A modified sample size method, discussed in the next section, can be used so that the observed confidence interval will be no wider than specified with some desired degree of assurance.

\section{Ensuring the Confidence Interval is Sufficiently Narrow With a Desired Degree of Assurance}

Recall that planning sample size so that the expected confidence interval width is sufficiently narrow does not imply that it will be sufficiently narrow in any particular study. The confidence interval width is a random variable because it is a function of the random statistic $R^{2}$, which is a function of the random data and the fixed design factors. A modified sample size procedure is developed that can be used in order to guarantee with some desired degree of assurance that the observed confidence interval for $\mathrm{P}^{2}$ will be no wider than desired. In order to carry out this modified method, the desired degree of assurance, denoted $\gamma$, must be specified, where $\gamma$ represents the desired probability of achieving a confidence interval no wider than desired. This procedure yields a modified sample size so that the obtained confidence interval will be no wider than $\omega$ with no less than $\gamma 100 \%$ assurance: $p(w \leq \omega) \geq \gamma$. For example, suppose one would like to have $99 \%$ assurance that the obtained $95 \%$ confidence interval will be no wider than .10 . In such a case $\gamma$ is set to .99 and $\omega$ is set to .10 , implying that an observed confidence interval for $\mathrm{P}^{2}$ will be wider than desired no more than $1 \%$ of the time. The way in which the modified sample size procedure works is quite involved and necessitates several steps. As before, no formal mathematical proof is known to exist, but an a priori Monte Carlo simulation study is developed later so that the exact sample size can be obtained.

Depending on the particular situation, obtaining an $R^{2}$ either larger or smaller than $\mathrm{E}\left[R^{2}\right]$, which is the value the standard sample size was based, will lead to a wider than desired confidence interval. For example, suppose sample size is based on $\mathrm{E}\left[R^{2}\right]=.60$, where $N=100, K=5$, and $1-\alpha=.95$. From Figure 1 it can be seen that any value of $R^{2}$ larger than .60 in this situation will lead to a $w<\omega$ (desirable). However, values of $R^{2}$ between .1676 and .6 will lead to a $w>\omega$ (not desirable), whereas values of $R^{2}$ less than .1676 will lead to a $w<\omega$ (desirable). This situation can be contrasted to one where sample size is based on $\mathrm{E}\left[R^{2}\right]=.20$, where $N=100, K=5$, and $1-\alpha=.95$. From Figure 1, it can be seen that any value of $R^{2}$ smaller than .20 in this situation will lead to a $w<\omega$ (desirable). However, values of $R^{2}$ between .2 and .5527 will lead to a $w>\omega$ (not desirable), whereas values of $R^{2}$ greater than .5527 will lead to a $w<\omega$ (desirable). Thus, depending on $\mathrm{E}\left[R^{2}\right]$ for the particular situation, obtaining an $R^{2}$ value either larger or smaller than $\mathrm{P}^{2}$ can lead to a wider than desired confidence interval. One thing not obvious from the figure is the probability that $R^{2}$ will be beyond the values that lead to larger 
or smaller than desired $w$ values (i.e., the sampling distribution of $R^{2}$ is not addressed in the figure). These issues, and another issue discussed momentarily, need to be addressed in order for the necessary sample size to be planned so that the $p(w \leq \omega) \geq \gamma$.

The starting point for the modified sample size, denoted $N_{\mathrm{M}}$, planning procedure is the original sample size based on the expected width being sufficiently narrow. Given the necessary sample size from the standard procedure, an upper and a lower $\gamma 100 \%$ one-sided confidence interval is formed using $\mathrm{E}\left[R^{2}\right]$ in place of $R^{2}$ (as was done in the standard confidence interval formation procedure section). The reason two $\gamma 100 \%$ one-sided confidence intervals are specified is to determine the $R^{2}$ value that will be exceeded $(1-\gamma) 100 \%$ of the time and the $R^{2}$ value that will exceed only $(1-\gamma) 100 \%$ of the distribution of $R^{2}$ values. These lower and upper confidence limits for $R^{2}$, denoted $\mathrm{P}_{\mathrm{L}^{*}}^{2}$ and $\mathrm{P}_{\mathrm{U}^{*}}^{2}$, are then used in the standard procedure as if they were the population values. There will then be two different sample sizes, one based on treating $\mathrm{P}_{\mathrm{L}^{*}}^{2}$ as if it were $\mathrm{P}^{2}$ and one based on treating $\mathrm{P}_{\mathrm{U}^{*}}^{2}$ as if it were $\mathrm{P}^{2}$. The larger of these two sample sizes is then taken as the preliminary value of the necessary sample size.

Ignoring a complication addressed momentarily, a discussion of the rationale for the approach thus far is given. Using $\mathrm{E}\left[R^{2}\right]$ in the two $\gamma 100 \%$ confidence intervals in place of $R^{2}$, the sampling distribution of $R^{2}$ will be less than $\mathrm{P}_{\mathrm{L}^{*}}^{2}$ $(1-\gamma) 100 \%$ of the time and greater than $\mathrm{P}_{\mathrm{U}^{*}}^{2}(1-\gamma) 100 \%$ of the time. The rationale for using $\mathrm{P}_{\mathrm{L}^{*}}^{2}$ and $\mathrm{P}_{\mathrm{U}^{*}}^{2}$ in place of $\mathrm{P}^{2}$ in the standard procedure is to find the value from the distribution of $R^{2}$ values that will divide the sampling distribution of $w$ values from those that are desirable (i.e., $w \leq \omega$ ) from those that are undesirable (i.e., $w>\omega$ ), while maintaining the probability of a $w \leq \omega$ at the specified level. The reason for using two one-sided $\gamma 100 \%$ confidence intervals is that depending on the situation, values larger than the confidence limit or values smaller than the limit will lead to confidence intervals wider than desired. Thus, values beyond one of the limits will lead to $R^{2}$ values that produce confidence intervals wider than desired $(1-\gamma) 100 \%$ of the time, but in any particular situation it is not known which limit. Thus, if the value of $R^{2}$ that leads to a $w$ larger than desired can be found (which is either $\mathrm{P}_{\mathrm{L}^{*}}^{2}$ or $\mathrm{P}_{\mathrm{U}^{*}}^{2}$ [momentarily ignoring the complication yet to be discussed]), then the standard sample size procedure can be based on $\mathrm{P}_{\mathrm{L}^{*}}^{2}$ or $\mathrm{P}_{\mathrm{U}^{*}}^{2}$ in place of $\mathrm{P}^{2}$. Doing so will lead to a sample size where no more than $(1-\gamma) 100 \%$ of the $w$ values are wider than $\omega$. Recall that this sample size is regarded as preliminary, as there is a potential complication that will arise in certain situations that is now addressed.

Because the relationship between $R^{2}$ and the confidence interval width is not monotonic (recall Figure 1), difficulties with the approach just presented can arise in a limited set of circumstances. Although one difficulty was in part addressed by using both the lower and the upper $\gamma 100 \%$ one-sided confidence 
limits in order to find the larger of the two sample sizes, in some situations values between the confidence limits will lead to wider than desired confidence intervals. When there are $R^{2}$ values contained within the two one-sided $\gamma 100 \%$ confidence interval limits that lead to a $w>\omega$, it is necessary to incorporate an additional step in the procedure. This additional step is so that no more than $(1-\gamma) 100 \%$ of the sampling distribution of $R^{2}$ is contained within the limits sample size is based.

Let $R_{\max (w)}^{2}$ be the $R^{2}$ value that leads to the maximum confidence interval width for a particular set of design factors. If $R_{\max (w)}^{2}$ is outside of (i.e., beyond) the confidence interval limits defined by the two one-sided $\gamma 100 \%$ confidence intervals (i.e., $R_{\max (w)}^{2} \notin\left[\mathrm{P}_{\mathrm{L}^{*}}^{2}, \mathrm{P}_{\mathrm{U}^{*}}^{2}\right]$ ), then no complication arises and the procedure stops by choosing the largest of the previously calculated sample sizes. However, if $R_{\max (w)}^{2}$ is contained within the interval defined by the limits of the two one-sided $\gamma 100 \%$ confidence intervals (i.e., $R_{\max (w)}^{2} \in\left[\mathrm{P}_{\mathrm{L}^{*}}^{2}, \mathrm{P}_{\mathrm{U}^{*}}^{2}\right]$ ), then an additional step is necessary. Although no known derivation exists for finding $R_{\max (w)}^{2}$ between two limits, it is easy to find $R_{\max (w)}^{2}$ with an optimization search routine. When $R_{\max (w)}^{2} \in\left[\mathrm{P}_{\mathrm{L}^{*}}^{2}, \mathrm{P}_{\mathrm{U}^{*}}^{2}\right]$, basing the sample size planning procedure on $R_{\max (w)}^{2}$ would lead to $100 \%$ of the confidence intervals being sufficiently narrow, as it would not be possible to obtain a confidence interval wider than $\omega$ when sample size is based on $R_{\max (w)}^{2}$. Because $\gamma$ would not generally be set to 1 , although doing so imposes no special difficulties in the present context, an additional step must be taken in order to obtain the modified sample size so that no less than $\gamma 100 \%$ of the confidence intervals are sufficiently narrow. The rationale for this additional step is to base the sample size procedure on the values of $R^{2}$ that bound the widest $(1-\gamma) 100 \%$ of the distribution of $w$. Doing so will lead to no more than $(1-\gamma) 100 \%$ of the confidence intervals being wider than $\omega$.

Although it may seem desirable at first to plan sample size so that $100 \%$ of the intervals are no wider than desired, doing so would tend to lead to a larger sample size than necessary whenever $\gamma<1$. An additional step of forming a two-sided $(1-\gamma) 100 \%$ confidence interval using $R_{\max (w)}^{2}$ as the point estimate is necessary for $(1-\gamma) 100 \%$ of the sampling distribution of the $w$ s to be larger than desired (and thus $\gamma 100 \%$ of the sampling distribution of the $w$ s smaller than desired). The limits of the $(1-\gamma) 100 \%$ two-sided confidence interval based on $R_{\max (w)}^{2}$ are then used in the standard procedure so that $\gamma 100 \%$ of the confidence intervals will be sufficiently narrow when $R_{\max (w)}^{2} \in\left[\mathrm{P}_{\mathrm{L}^{*}}^{2}, \mathrm{P}_{\mathrm{U}^{*}}^{2}\right]$. Formation of the $(1-\gamma) 100 \%$ two-sided confidence interval is to exclude the $(1-\gamma) 100 \%$ of the sampling distribution of $R^{2}$ values that would yield $w$ values smaller than desired (so that no less than $\gamma 100 \%$ of the $w$ values will be less than $\omega$ ). The larger of the four sample sizes based on the four confidence limits substituted into the standard procedure, two from this additional step (when necessary) and 
two from the two one-sided confidence limits in the first step of the modified sample size procedure, is the necessary sample size. Necessary sample size cannot be based solely on the limits at this step because these limits can lead to a smaller sample size than necessary when based on one or both of the limits from the two one-sided $\gamma 100 \%$ confidence limits. That is, the lower or upper limit from the $(1-\gamma) 100 \%$ two-sided confidence interval based on $R_{\max (w)}^{2}$ can be beyond the upper or lower limits of the confidence intervals based on $\mathrm{P}_{\mathrm{L}^{*}}^{2}$ or $\mathrm{P}_{\mathrm{U}^{*}}^{2}$, respectively.

A step-by-step summary of the procedure is provided.

Step 1: Based on the necessary sample size from the standard procedure, two one-sided $\gamma 100$ confidence intervals are calculated.

Step 2: The limits from Step 1 are used in the standard procedure as if they were $\mathrm{P}^{2}$. The larger of the two sample sizes is regarded as the preliminary value of necessary sample size.

Step 3: The value of $R_{\max (w)}^{2}$ that leads to the maximum confidence interval width is found.

Step 4: If $R_{\max (w)}^{2}$ is not contained within the limits of the two one-sided $\gamma 100 \%$ confidence intervals, no additional steps are necessary and sample size is set to the preliminary sample size value in Step 2.

Step 5 [if necessary]: If $R_{\max (w)}^{2}$ is contained within the limits of the two one-sided $(1-\gamma) 100 \%$ confidence intervals, then the limits of the twosided $(1-\gamma) 100 \%$ confidence interval based on $R_{\max (w)}^{2}$ are used in the standard procedure as if they were $\mathrm{P}^{2}$.

Step 6 [if necessary]: The largest sample size from the contending sample sizes (from Step 2 and from Step 5) is taken as the necessary value.

The steps above are used to find the value of $\mathrm{E}\left[R^{2}\right]$ that when substituted for $\mathrm{P}^{2}$ in the standard procedure will lead to no less than $\gamma 100 \%$ of the confidence intervals being sufficiently narrow. As discussed momentarily, a Monte Carlo simulation study revealed that procedures perform very well but not perfectly. The next section discusses a computationally intense a priori Monte Carlo simulation that can be used to obtain the exact sample size in any situation of interest.

\section{Obtaining the Exact Sample Size}

This section discusses a computationally intense approach that leads to the exact value of sample size in any condition, provided certain assumptions are met. As noted, the methods previously discussed are approximate, although as shown later they tend to yield very close approximations. Because a mathematical proof 
that would always lead to the exact sample size in the context of AIPE for $\mathrm{P}^{2}$ has not been established, a general principle of sample size planning is applied: the exact value of necessary sample size can be planned in any situation under any goal with the appropriate use of computationally intense procedures. The benefit of a computationally intense approach, known as an a priori Monte Carlo simulation, is that by designing the appropriate a priori Monte Carlo simulation, exact sample size can be planned in any situation of interest. The idea is to generate data that conforms to the population of interest, which requires the assumption that what is true in the population is represented in the simulation procedure (this is also implicit for analytic approaches to sample size planning), and determine the minimum sample size so that the particular goal is satisfied. One goal might be rejecting the null hypothesis that a particular effect equals zero with no less than .85 probability. Another goal might be obtaining a $95 \%$ confidence interval for some population effect size less than $\zeta$ units with .99 probability, where $\zeta$ is the desired width in some situation. Yet another goal might be a combination where the null hypothesis is rejected with no less than .85 probability and the $95 \%$ confidence interval is sufficiently narrow with no less than .99 probability. The benefit of the computationally intense approach is that it can be used for any procedure, and existing analytic work on sample size planning need not exist-or if it does it need not be exact. In order for the sample size to literally be exact, (a) all necessary assumptions of the model must be correct, (b) all necessary parameters must be correctly specified, and (c) the number of replications needs to tend toward infinity.

Muthén and Muthén (2002) discuss a similar a priori Monte Carlo simulation study in the context of factor analysis and structural equation modeling (see also Satorra \& Saris, 1985). M'Lan, Joseph, and Wolfson (2006) apply an a priori Monte Carlo study for sufficiently narrow confidence interval estimates for casecontrol studies in a Bayesian framework (see also Joseph, Berger, \& Bélisle, 1995; Wang \& Gelfand, 2002). In general, Monte Carlo simulation studies are used to evaluate properties of some aspect of the distribution of some effect, often to assess various properties of a procedure or to evaluate robustness when the model assumptions are violated. In an a priori Monte Carlo study, however, what is of interest is the properties of statistics, and functions of them, so that the information can be used to plan a study in order to accomplish some desired goal.

In the context of sample size planning for a desired power for the test of $\mathrm{P}^{2}=0$, O'Brien \& Muller (1993) base sample size planning on models with fixed regressors with the intended use being models with random regressors. They state that "because the population parameters are conjectures or estimates, strict numerical accuracy of the power computations is usually not critical" (p. 23). Although sample size will likely never be perfect because the population 
parameter(s) are almost certainly not known exactly, it is also desirable for procedures to always yield the exact answer if the parameters were known perfectly. The a priori Monte Carlo simulation generates data conforming to the $K$ and $\mathrm{P}^{2}$ situation specified for multivariate normal data and calculates the confidence interval for $\mathrm{P}^{2}$. The process is replicated a large number of times (e.g., 10,000). If $\gamma_{\mathrm{E}}<\gamma$, sample size is increased by one and the simulation again generates a large number of replications from the specified situation, where $\gamma_{E}$ is the empirical value of $\gamma$ from the specified condition. ${ }^{5}$ Conversely, if $\gamma_{E}>\gamma$, the procedure recommended sample size is reduced by 1 and the simulation again generates a large number of replications from the specified situation. Successive sample sizes are found where $N_{(i)}$ leads to $\gamma_{\mathrm{E}}<\gamma$ and $N_{(i+1)}$ lead to $\gamma_{\mathrm{E}} \geq \gamma$ (or vice versa). The value of $N_{(i+1)}$ is then set to necessary sample size (or vice versa) because it is the minimum value of sample size where the specifications are satisfied. Provided the number of replications is large enough, the procedure will return the exact value of sample size. Although this Monte Carlo sample size verification feature was discussed in the context of sample size planning when $\gamma$ is specified, it is equally applicable for planning the expected confidence interval width or the median confidence interval width instead of the expected width (i.e., the mean) is sufficiently narrow.

\section{EFFECTIVENESS OF THE PROCEDURES}

A large scale Monte Carlo simulation study was conducted where $K(2,5$, $\& 10), \mathrm{P}^{2}(.10$ to .90 by .1$), \omega(.05 \& .10$ to .40 by .10$)$, and $\gamma$ (expected width, .85, \& .99) were manipulated, leading to a $3 \times 9 \times 5 \times 3$ factorial design (405 conditions) in the case of random regressors. The conditions examined are thought to be realistic for most applications of AIPE for $\mathrm{P}^{2}$ within the BEMS sciences. For each of the conditions evaluated, multivariate normal data were generated that conformed to the specific conditions $\left(N, K, \& \mathrm{P}^{2}\right)$ in order to obtain a sample $R^{2}$ value and form a confidence interval for $\mathrm{P}^{2}$ in accord with the method of confidence interval formation for random regressors (using the ci.R2() MBESS function, as discussed later). All of the assumptions were satisfied and each set of results is based on 10,000 replications. The specific results are reported for $K=2,5$, and 10 in subtable A (the upper table) of Tables 1, 2, and 3, respectively. Subtable A within Tables 1, 2, and 3 reports

\footnotetext{
${ }^{5}$ Actually, it is desirable to first use a much smaller number of replications (e.g., 1,000) to home in on necessary sample size. After an approximate sample size is determined in the manner discussed, then a large number of replications (e.g., 10,000) is used to find the exact value.
} 
TABLE 1

Results of the Monte Carlo Simulation Study Based on the Standard Procedure (A) and the Modified Procedure Where $\gamma=.85$ (B) for $K=2$

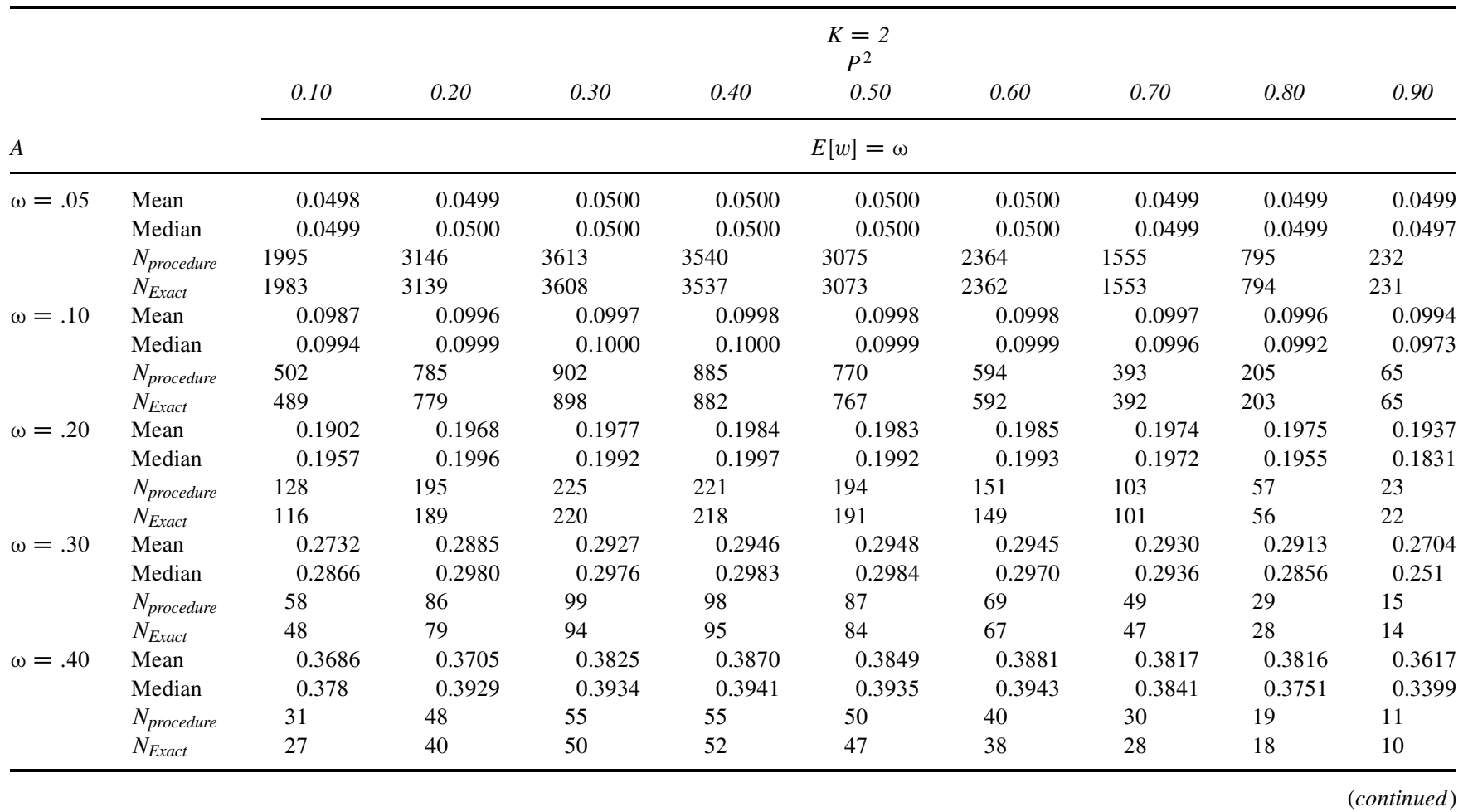


TABLE 1

(Continued)

\begin{tabular}{|c|c|c|c|c|c|c|c|c|c|c|}
\hline \multirow[b]{2}{*}{$B$} & & \multicolumn{9}{|c|}{$\begin{array}{c}K=2 \\
P^{2}\end{array}$} \\
\hline & & & & & & $\gamma=.85$ & & & & \\
\hline \multirow[t]{3}{*}{$\omega=.05$} & $\% w<\omega$ & 0.8508 & 0.8539 & 0.8589 & 0.8636 & 0.8562 & 0.8598 & 0.864 & 0.8612 & 0.8788 \\
\hline & $N_{\text {procedure }}$ & 2182 & 3241 & 3631 & 3576 & 3155 & 2468 & 1663 & 887 & 289 \\
\hline & $N_{\text {Exact }}$ & 2184 & 3244 & 3631 & 3576 & 3152 & 2464 & 1658 & 882 & 284 \\
\hline \multirow[t]{3}{*}{$\omega=.10$} & $\% w<\omega$ & 0.8493 & 0.8466 & 0.9263 & 0.8711 & 0.8645 & 0.8658 & 0.8694 & 0.8806 & 0.8969 \\
\hline & $N_{\text {procedure }}$ & 587 & 829 & 909 & 901 & 809 & 645 & 447 & 251 & 94 \\
\hline & $N_{\text {Exact }}$ & 590 & 830 & 909 & 900 & 807 & 643 & 443 & 246 & 89 \\
\hline \multirow[t]{3}{*}{$\omega=.20$} & $\% w<\omega$ & 0.765 & 0.8431 & 1 & 1 & 0.889 & 0.8842 & 0.8835 & 0.8977 & 0.9521 \\
\hline & $N_{\text {procedure }}$ & 164 & 214 & 227 & 227 & 212 & 176 & 129 & 80 & 37 \\
\hline & $N_{\text {Exact }}$ & 165 & 215 & 227 & 226 & 210 & 174 & 126 & 76 & 33 \\
\hline \multirow[t]{3}{*}{$\omega=.30$} & $\% w<\omega$ & 0.7313 & 0.8511 & 1 & 0.7373 & 0.8903 & 0.8927 & 0.9062 & 0.9114 & 0.965 \\
\hline & $N_{\text {procedure }}$ & 79 & 97 & 100 & 99 & 97 & 85 & 66 & 44 & 24 \\
\hline & $N_{\text {Exact }}$ & 80 & 97 & 100 & 100 & 97 & 83 & 63 & 41 & 20 \\
\hline \multirow[t]{3}{*}{$\omega=.40$} & $\% w<\omega$ & 0.7488 & 0.7997 & 1 & 1 & 1 & 0.9065 & 0.9191 & 0.9541 & 0.973 \\
\hline & $N_{\text {procedure }}$ & 47 & 55 & 56 & 56 & 56 & 51 & 42 & 30 & 18 \\
\hline & $N_{\text {Exact }}$ & 48 & 60 & 56 & 56 & 55 & 49 & 39 & 27 & 15 \\
\hline
\end{tabular}

Note. $\quad \mathrm{P}^{2}$ is the population squared multiple correlation coefficient, $\omega$ is the desired confidence interval width, $w$ is the observed confidence interval width, $N_{\text {Procedure }}$ is the procedure implied sample size, $N_{\text {Exact }}$ is the sample size from the a priori Monte Carlo simulation, and $\gamma$ is the assurance parameter. 
TABLE 2

Results of the Monte Carlo Simulation Study Based on the Standard Procedure (A) and the Modified Procedure Where $\gamma=.85$ (B) for $K=5$

\begin{tabular}{|c|c|c|c|c|c|c|c|c|c|c|}
\hline \multirow[b]{2}{*}{$A$} & & \multicolumn{9}{|c|}{$\begin{array}{c}K=5 \\
P^{2}\end{array}$} \\
\hline & & & & & & $E[w]=\omega$ & & & & \\
\hline \multirow[t]{4}{*}{$\omega=.05$} & Mean & 0.0499 & 0.0500 & 0.0500 & 0.0500 & 0.0500 & 0.0500 & 0.0500 & 0.0499 & 0.0498 \\
\hline & Median & 0.0499 & 0.0500 & 0.0500 & 0.0500 & 0.0500 & 0.0500 & 0.0500 & 0.0499 & 0.0495 \\
\hline & $N_{\text {procedure }}$ & 2010 & 3153 & 3618 & 3544 & 3078 & 2366 & 1557 & 797 & 233 \\
\hline & $N_{\text {Exact }}$ & 2001 & 3147 & 3613 & 3541 & 3075 & 2365 & 1555 & 796 & 233 \\
\hline \multirow[t]{4}{*}{$\omega=.10$} & Mean & 0.0988 & 0.0996 & 0.0997 & 0.0998 & 0.0998 & 0.0998 & 0.0998 & 0.0995 & 0.0995 \\
\hline & Median & 0.0995 & 0.0999 & 0.1000 & 0.1000 & 0.0999 & 0.0999 & 0.0997 & 0.0992 & 0.0972 \\
\hline & $N_{\text {procedure }}$ & 516 & 793 & 907 & 889 & 773 & 596 & 395 & 207 & 67 \\
\hline & $N_{\text {Exact }}$ & 504 & 786 & 903 & 886 & 770 & 595 & 395 & 206 & 67 \\
\hline \multirow[t]{4}{*}{$\omega=.20$} & Mean & 0.1905 & 0.1967 & 0.1978 & 0.1984 & 0.1984 & 0.1986 & 0.1978 & 0.1970 & 0.1937 \\
\hline & Median & 0.1968 & 0.1994 & 0.1992 & 0.1998 & 0.1993 & 0.1993 & 0.1975 & 0.1943 & 0.1832 \\
\hline & $N_{\text {procedure }}$ & 140 & 203 & 230 & 225 & 197 & 154 & 105 & 59 & 25 \\
\hline & $N_{\text {Exact }}$ & 127 & 196 & 225 & 222 & 195 & 152 & 103 & 59 & 25 \\
\hline \multirow[t]{4}{*}{$\omega=.30$} & Mean & 0.2761 & 0.2890 & 0.2930 & 0.2946 & 0.2951 & 0.2936 & 0.2942 & 0.2877 & 0.2811 \\
\hline & Median & 0.2873 & 0.2984 & 0.2972 & 0.2984 & 0.2986 & 0.2958 & 0.2937 & 0.2812 & 0.2605 \\
\hline & $N_{\text {procedure }}$ & 65 & 93 & 104 & 102 & 90 & 72 & 51 & 32 & 17 \\
\hline & $N_{\text {Exact }}$ & 56 & 87 & 100 & 99 & 88 & 70 & 50 & 31 & 16 \\
\hline \multirow[t]{4}{*}{$\omega=.40$} & Mean & 0.3778 & 0.35 & 0.3840 & 0.3876 & 0.3879 & 0.3883 & 0.3883 & 0.3784 & 0.3519 \\
\hline & Median & 0.3875 & 0.3681 & 0.3938 & 0.3952 & 0.3963 & 0.3936 & 0.3911 & 0.3716 & 0.3214 \\
\hline & $N_{\text {procedure }}$ & 35 & 55 & 60 & 59 & 53 & 43 & 32 & 22 & 14 \\
\hline & $N_{\text {Exact }}$ & 32 & 46 & 55 & 56 & 51 & 41 & 31 & 21 & 13 \\
\hline
\end{tabular}


TABLE 2

(Continued)

\begin{tabular}{|c|c|c|c|c|c|c|c|c|c|c|}
\hline \multirow[b]{2}{*}{$B$} & & \multicolumn{9}{|c|}{$\begin{array}{c}K=2 \\
P^{2}\end{array}$} \\
\hline & & & & & & $\gamma=.85$ & & & & \\
\hline \multirow[t]{3}{*}{$\omega=.05$} & $\% w<\omega$ & 0.8265 & 0.8335 & 0.851 & 0.8627 & 0.8668 & 0.8656 & 0.8685 & 0.8712 & 0.895 \\
\hline & $N_{\text {procedure }}$ & 2178 & 3243 & 3635 & 3581 & 3160 & 2474 & 1669 & 894 & 295 \\
\hline & $N_{\text {Exact }}$ & 2201 & 3249 & 3636 & 3579 & 3155 & 2467 & 1660 & 884 & 285 \\
\hline \multirow[t]{3}{*}{$\omega=.10$} & $\% w<\omega$ & 0.8066 & 0.8194 & 1 & 0.8823 & 0.8828 & 0.8897 & 0.8902 & 0.8989 & 0.921 \\
\hline & $N_{\text {procedure }}$ & 585 & 832 & 914 & 906 & 815 & 652 & 454 & 258 & 100 \\
\hline & $N_{\text {Exact }}$ & 601 & 836 & 913 & 905 & 810 & 646 & 446 & 248 & 91 \\
\hline \multirow[t]{3}{*}{$\omega=.20$} & $\% w<\omega$ & 0.765 & 0.7918 & 1 & 1 & 0.9037 & 0.9076 & 0.9108 & 0.9299 & 0.9521 \\
\hline & $N_{\text {procedure }}$ & 164 & 217 & 231 & 231 & 217 & 182 & 135 & 86 & 43 \\
\hline & $N_{\text {Exact }}$ & 174 & 221 & 231 & 231 & 214 & 176 & 128 & 79 & 35 \\
\hline \multirow[t]{3}{*}{$\omega=.30$} & $\% w<\omega$ & 0.7313 & 0.7566 & 1 & 0.6813 & 0.9508 & 0.9229 & 0.928 & 0.946 & 0.965 \\
\hline & $N_{\text {procedure }}$ & 80 & 100 & 105 & 103 & 103 & 90 & 71 & 50 & 29 \\
\hline & $N_{\text {Exact }}$ & 88 & 103 & 105 & 105 & 100 & 86 & 65 & 43 & 23 \\
\hline \multirow[t]{3}{*}{$\omega=.40$} & $\% w<\omega$ & 0.7488 & 0.7997 & 1 & 1 & 1 & 0.9388 & 0.948 & 0.9541 & 0.973 \\
\hline & $N_{\text {procedure }}$ & 50 & 59 & 61 & 61 & 61 & 56 & 47 & 35 & 23 \\
\hline & $N_{\text {Exact }}$ & 55 & 60 & 60 & 60 & 59 & 53 & 43 & 29 & 18 \\
\hline
\end{tabular}

Note. $\quad \mathrm{P}^{2}$ is the population squared multiple correlation coefficient, $\omega$ is the desired confidence interval width, $w$ is the observed confidence interval width, $N_{\text {Procedure }}$ is the procedure implied sample size, $N_{\text {Exact }}$ is the sample size from the a priori Monte Carlo simulation, and $\gamma$ is the assurance parameter. 
TABLE 3

Results of the Monte Carlo Simulation Study Based on the Standard Procedure (A) and the Modified Procedure Where $\gamma=.85$ (B) for $K=10$

\begin{tabular}{|c|c|c|c|c|c|c|c|c|c|c|}
\hline \multirow[b]{2}{*}{$A$} & & \multicolumn{9}{|c|}{$\begin{array}{c}K=10 \\
P^{2}\end{array}$} \\
\hline & & & & & & $E[w]=\omega$ & & & & \\
\hline \multirow[t]{4}{*}{$\omega=.05$} & Mean & 0.0499 & 0.0499 & 0.0500 & 0.0500 & 0.0500 & 0.0500 & 0.0500 & 0.0500 & 0.0499 \\
\hline & Median & 0.0500 & 0.0500 & 0.0500 & 0.0500 & 0.0500 & 0.0500 & 0.0500 & 0.0499 & 0.0497 \\
\hline & $N_{\text {procedure }}$ & 2034 & 3166 & 3626 & 3550 & 3083 & 2371 & 1560 & 800 & 236 \\
\hline & $N_{\text {Exact }}$ & 2026 & 3159 & 3622 & 3547 & 3081 & 2371 & 1560 & 799 & 237 \\
\hline \multirow[t]{4}{*}{$\omega=.10$} & Mean & 0.0988 & 0.0995 & 0.0997 & 0.0998 & 0.0998 & 0.0997 & 0.0997 & 0.0995 & 0.0999 \\
\hline & Median & 0.0994 & 0.0999 & 0.0999 & 0.1000 & 0.1000 & 0.0998 & 0.0996 & 0.0990 & 0.0980 \\
\hline & $N_{\text {procedure }}$ & 538 & 805 & 916 & 895 & 778 & 601 & 399 & 210 & 70 \\
\hline & $N_{\text {Exact }}$ & 528 & 799 & 911 & 892 & 776 & 599 & 398 & 209 & 70 \\
\hline \multirow[t]{4}{*}{$\omega=.20$} & Mean & 0.1896 & 0.1970 & 0.1979 & 0.1986 & 0.1986 & 0.1985 & 0.1979 & 0.1969 & 0.1945 \\
\hline & Median & 0.1974 & 0.1997 & 0.1992 & 0.1999 & 0.1996 & 0.1992 & 0.1973 & 0.1945 & 0.1848 \\
\hline & $N_{\text {procedure }}$ & 158 & 214 & 238 & 231 & 202 & 158 & 109 & 63 & 29 \\
\hline & $N_{\text {Exact }}$ & 127 & 208 & 234 & 228 & 200 & 157 & 107 & 62 & 25 \\
\hline \multirow[t]{4}{*}{$\omega=.30$} & Mean & 0.2811 & 0.2892 & 0.2938 & 0.2940 & 0.2945 & 0.2935 & 0.2957 & 0.2902 & 0.2535 \\
\hline & Median & 0.292 & 0.2986 & 0.2973 & 0.2980 & 0.2979 & 0.2950 & 0.2951 & 0.2846 & 0.2367 \\
\hline & $N_{\text {procedure }}$ & 74 & 104 & 112 & 109 & 96 & 77 & 55 & 36 & 23 \\
\hline & $N_{\text {Exact }}$ & 56 & 97 & 108 & 106 & 94 & 75 & 54 & 35 & 16 \\
\hline \multirow[t]{4}{*}{$\omega=.40$} & Mean & 0.3795 & 0.3651 & 0.3840 & 0.3871 & 0.3867 & 0.3894 & 0.3863 & 0.3733 & 0.2542 \\
\hline & Median & 0.3891 & 0.395 & 0.3939 & 0.3953 & 0.3938 & 0.3937 & 0.3851 & 0.3656 & 0.2384 \\
\hline & $N_{\text {procedure }}$ & 42 & 65 & 68 & 66 & 59 & 48 & 37 & 27 & 23 \\
\hline & $N_{\text {Exact }}$ & 32 & 46 & 63 & 63 & 56 & 46 & 36 & 21 & 13 \\
\hline
\end{tabular}


TABLE 3

(Continued)

\begin{tabular}{|c|c|c|c|c|c|c|c|c|c|c|}
\hline \multirow[b]{2}{*}{$B$} & & \multicolumn{9}{|c|}{$\begin{array}{c}K=2 \\
P^{2}\end{array}$} \\
\hline & & & & & & $\gamma=.85$ & & & & \\
\hline \multirow[t]{3}{*}{$\omega=.05$} & $\% w<\omega$ & 0.7812 & 0.8094 & 0.8269 & 0.8777 & 0.8873 & 0.8779 & 0.8893 & 0.8978 & 0.9168 \\
\hline & $N_{\text {procedure }}$ & 2172 & 3246 & 3642 & 3589 & 3170 & 2485 & 1681 & 905 & 306 \\
\hline & $N_{\text {Exact }}$ & 2218 & 3261 & 3644 & 3586 & 3161 & 2471 & 1664 & 889 & 288 \\
\hline \multirow[t]{3}{*}{$\omega=.10$} & $\% w<\omega$ & 0.7124 & 0.7638 & 0.9128 & 0.9064 & 0.9008 & 0.902 & 0.9153 & 0.9217 & 0.9554 \\
\hline & $N_{\text {procedure }}$ & 581 & 836 & 921 & 914 & 824 & 662 & 464 & 268 & 110 \\
\hline & $N_{\text {Exact }}$ & 619 & 849 & 921 & 911 & 816 & 650 & 450 & 252 & 94 \\
\hline \multirow[t]{3}{*}{$\omega=.20$} & $\% w<\omega$ & - & 0.6713 & 1 & 1 & 0.9413 & 0.946 & 0.9464 & 0.9596 & 0.9757 \\
\hline & $N_{\text {procedure }}$ & 163 & 222 & 239 & 239 & 226 & 192 & 145 & 95 & 51 \\
\hline & $N_{\text {Exact }}$ & 174 & 231 & 239 & 238 & 219 & 182 & 132 & 81 & 35 \\
\hline \multirow[t]{3}{*}{$\omega=.30$} & $\% w<\omega$ & - & 0.6081 & 1 & 0.6629 & 0.9714 & 0.9708 & 0.9744 & 0.9803 & 0.9908 \\
\hline & $N_{\text {procedure }}$ & - & 106 & 112 & 110 & 111 & 100 & 81 & 59 & 37 \\
\hline & $N_{\text {Exact }}$ & 88 & 111 & 112 & 112 & 106 & 91 & 70 & 47 & 23 \\
\hline \multirow[t]{3}{*}{$\omega=.40$} & $\% w<\omega$ & - & - & 1 & 1 & 1 & 0.9797 & 0.982 & 0.9884 & 0.992 \\
\hline & $N_{\text {procedure }}$ & - & - & 68 & 68 & 68 & 65 & 56 & 44 & 30 \\
\hline & $N_{\text {Exact }}$ & 55 & 60 & 68 & 68 & 66 & 58 & 47 & 29 & 18 \\
\hline
\end{tabular}

Note. $\mathrm{P}^{2}$ is the population squared multiple correlation coefficient, $\omega$ is the desired confidence interval width, $w$ is the observed confidence interval width, $N_{\text {Procedure }}$ is the procedure implied sample size, $N_{\text {Exact }}$ is the sample size from the a priori Monte Carlo simulation, and $\gamma$ is the assurance parameter. 
the mean and median confidence interval widths, the procedure implied sample size $\left(N_{\text {Procedure }}\right)$ and the exact approach to sample size planning as determined by the a priori Monte Carlo procedure (denoted $N_{\text {Exact }}$, which itself is based on 10,000 replications).

For the conditions where the expected widths were examined using the originally proposed procedure, the mean of the discrepancy between the mean confidence interval width and the desired value was $-.0075,-.0075$, and -.0099 for $K=2,5$, and 10, respectively, with corresponding standard deviations of .0101, .0113, and .023, respectively. Examining the discrepancy between procedure implied sample size and the exact sample size as determined from the a priori Monte Carlo simulation procedure reveals that the mean sample size discrepancy is $3.71,3.36$, and 4.71 for $K=2,5$, and 10 , respectively, with corresponding standard deviations of $3.15,3.16$, and 5.79 , respectively.

Conditions where the $w$ s were systematically too small tended to be in conditions where the desired width was very large. In the case of the largest discrepancy between the specified and observed $\omega$, the mean and median $w$ was 0.2542 and 0.2384 , respectively, for the case where $\omega=.40, \mathrm{P}^{2}=.90$, and $K=10$. In this worst case, the procedure implied sample size was 23 , whereas the exact sample size is 13 . Sample size values are small when $\omega$ is large, implying a large sampling variability of $R^{2}$. What tends to happen for relatively large values of $\omega$ coupled with very small or very large values of $\mathrm{P}^{2}$ is that the procedure implied sample size is small and the confidence bound of 0 or 1 is reached with a nontrivial probability, and the confidence interval is smaller than it otherwise would have been if $\mathrm{P}^{2}$ were not bounded at 0 and 1 , due to the necessary truncation at the lower or upper (as is the case in this condition) confidence bound of the confidence interval procedure. The procedure does not consider truncated confidence intervals but the a priori Monte Carlo simulation study does, which is why it returns the better estimate of sample size in cases of a nontrivial amount of truncation. Otherwise, the closeness of the mean and median $w$ to $\omega$ was excellent and the procedure implied sample size did not generally differ considerably from the exact sample size procedure. Examination of Tables 1, 2, and 3 show that the method performed less than optimally generally when confidence intervals widths were large. Such cases tended to lead to relatively small sample sizes and large variability of $R^{2}$, which yielded a nontrivial amount of confidence interval truncation at either 0 or 1 . Many such situations are not likely to be of interest in the majority of applied research.

The pattern of results for $\gamma=.85$ is very similar to the case where $\gamma=$ .99 , and only the results for $\gamma=.85$ are reported and discussed for space considerations. The specific results are reported for $K=2,5$, and 10 in subtable B (the lower table) of Tables 1, 2, and 3. Four conditions when $K=10$ were not implementable (specifically $\mathrm{P}^{2}=.10$ for $\omega=.20, .30, \& .40$ as well as $\mathrm{P}^{2}=.20$ for $\omega=.40$ ) because the desired confidence interval width was too 
large given the size of $\mathrm{P}^{2}$ and number of regressors. In attempting to implement the modified sample size procedure as discussed, an intermediate confidence interval ranged from 0 to 1 and thus subsumed the entire range of allowable values for $\mathrm{P}^{2}$, which did not render subsequent steps possible. Such a failure of the procedure will occur only rarely, as it will not be likely researchers will plan sample sizes for very wide confidence intervals for very small (or very large) values of $\mathrm{P}^{2}$, coupled with a large number of predictors.

Examining the discrepancy between the procedure implied sample size and the exact sample size, as determined from the a priori Monte Carlo simulation study, reveals that the mean sample size discrepancy is $1.38,1.76$, and 3.86 for $K=2,5$, and 10 , respectively, with corresponding standard deviations of 2.44 , 6.79 , and 13.51, respectively. The worst case in terms of the raw discrepancy between $\gamma_{\mathrm{E}}$ and $\gamma$ was for $K=10, \mathrm{P}^{2}=.20$, and $\omega=.30$, where only $60.81 \%$ of the confidence intervals were sufficiently narrow, whereas $85 \%$ of the confidence intervals should have been given $\gamma$ was set to .85 . The sample sized used, as suggested by the procedure, was 106. A follow-up Monte Carlo simulation study using a sample size of 107, 108, 109, 110, and 111 showed that $64.16 \%, 68.85 \%, 73.90 \%, 80.10 \%$, and $86.13 \%$ of the confidence intervals were sufficiently narrow at those sample sizes, respectively. The exact sample size in this condition is thus 111 because 111 is the minimum value of sample size where $\gamma_{\mathrm{E}} \geq \gamma$. Thus, in the worse raw discrepancy between $\gamma_{\mathrm{E}}$ and $\gamma$ (a $85-60.81=24.19$ discrepancy in percentages), the procedure yielded a necessary sample size that was too small by less than $5 \%$.

Rather than observing a smaller than desired $\gamma_{\mathrm{E}}$ (i.e., less than $85 \%$ of the confidence intervals being sufficiently narrow), in some situations there was a larger than desired $\gamma_{\mathrm{E}}$ (i.e., more than $85 \%$ of the confidence intervals being sufficiently narrow). For example, examination of the condition where $\mathrm{P}^{2}=.20$, $\omega=.20$, and $K=10$ shows that $100 \%$ of the confidence intervals were sufficiently narrow for $N=239$ (the procedure implied sample size). However, a Monte Carlo simulation study shows that by reducing the sample size by 1 (to $N=238$ ) leads to only $78.56 \%$ of the confidence intervals being sufficiently narrow. Thus, the sample size of 239 is correct because it is the minimal value of sample size that leads to no less than $\gamma 100 \%$ of the confidence intervals being sufficiently narrow. This is in no way a deficiency of the proposed methods, but rather it is a property of confidence intervals for $\mathrm{P}^{2}$ because of the effect a small change in $N$ can have on the confidence interval properties. ${ }^{6}$ Of course, not all

\footnotetext{
${ }^{6}$ Similar issues also arise and have been reported in the context of AIPE for the standardized mean difference, where small increase in $N$ can have a large impact on $\gamma_{E}$ (elaboration on this issue is given in Kelley \& Rausch, 2006). Although not often discussed, similar issues as discussed here occur in power analysis, where increasing sample size by whole numbers almost always leads to an empirical power greater than the desired power.
} 
of the conditions where $\gamma_{\mathrm{E}}>\gamma$ yield the proper sample size, which can be seen by looking at the tables on a case-by-case basis. Nevertheless, from the summary statistics provided the proposed procedure is quite successful at recovering the proper sample size. However, given that the a priori Monte Carlo simulation study can be implemented, there are no practical problems for planning the exact sample size in any condition.

By its very nature, the a priori Monte Carlo method discussed yields the exact results, provided the number of replications is sufficiently large, the value of $\mathrm{P}^{2}$ has been correctly specified, and all model assumptions hold. This approach literally uses a Monte Carlo simulation study to determine at what sample size the specified goals are achieved. The minimum value of sample size where the specified goal is realized is set to the necessary sample size. The way in which the a priori Monte Carlo approach could be evaluated is with a Monte Carlo simulation study. Because the method itself is a Monte Carlo simulation, the results are literally the same. The difference between the a priori Monte Carlo simulation study and the (standard) Monte Carlo simulation study is that the a priori Monte Carlo study is used to plan a research study, whereas the standard Monte Carlo simulation study is used to evaluate the effectiveness of a method under a specified set of conditions. Evaluating the set of conditions with a Monte Carlo study using sample size as obtained from the a priori Monte Carlo simulation study thus leads to the conclusion that is already known: the a priori Monte Carlo procedure selects the sample size that yields the exact value of sample size.

\section{TABLES OF NECESSARY SAMPLE SIZE}

Although determining sample size so that sufficiently narrow confidence intervals can be obtained in the context of the multiple correlation coefficient with ease using MBESS (as discussed in the next section), the necessary sample sizes for a variety of conditions are provided. These tables are not meant to supplant the use of the methods developed or the computer routines provided, but rather they are designed so that researchers can better understand the nonlinear relationship that exists between the planned sample size conditional on $\mathrm{P}^{2}$, the $\omega$, $K$, and $\gamma$ as well as to use the tables for sample size planning when appropriate. Table 4 provides the necessary sample size in order for the expected width to be sufficiently narrow for 2,5 , and 10 regressors. Table 5 provides the necessary sample size in order for there to be a degree of assurance of .99 that the obtained confidence interval will be sufficiently narrow for the same conditions given in Table 4. 
Necessary Sample Size per Group for 95\% Confidence Intervals for the Population Squared Multiple Correlation Coefficient for Selected Situations When the Number of Regressors Equals 2, 5, and 10 for the Expected Width to Equal the Desired Width

\begin{tabular}{|c|c|c|c|c|c|c|c|c|c|c|c|c|c|c|c|c|c|c|c|}
\hline \multirow[b]{2}{*}{$\omega$} & \multicolumn{19}{|c|}{$P^{2}$} \\
\hline & 0.05 & 0.10 & 0.15 & 0.20 & 0.25 & 0.30 & 0.35 & 0.40 & 0.45 & 0.50 & 0.55 & 0.60 & 0.65 & 0.70 & 0.75 & 0.80 & 0.85 & 0.90 & 0.95 \\
\hline \multicolumn{20}{|c|}{$K=2$} \\
\hline 0.05 & 1106 & 1983 & 2657 & 3139 & 3450 & 3608 & 3631 & 3537 & 3345 & 3073 & 2740 & 2362 & 1961 & 1553 & 1159 & 794 & 478 & 231 & 69 \\
\hline 0.10 & 273 & 489 & 658 & 779 & 857 & 898 & 904 & 882 & 835 & 767 & 685 & 592 & 493 & 392 & 294 & 203 & 126 & 65 & 24 \\
\hline 0.15 & 120 & 214 & 288 & 342 & 377 & 396 & 399 & 390 & 370 & 341 & 305 & 264 & 221 & 177 & 134 & 94 & 60 & 33 & 15 \\
\hline 0.20 & 70 & 115 & 158 & 189 & 210 & 220 & 223 & 218 & 207 & 191 & 171 & 149 & 126 & 101 & 77 & 56 & 37 & 22 & 12 \\
\hline 0.25 & 47 & 71 & 98 & 118 & 131 & 139 & 141 & 138 & 132 & 122 & 110 & 96 & 81 & 66 & 51 & 38 & 26 & 17 & 10 \\
\hline 0.30 & 35 & 48 & 65 & 79 & 89 & 94 & 96 & 95 & 91 & 84 & 76 & 67 & 58 & 47 & 37 & 28 & 20 & 14 & 9 \\
\hline 0.35 & 27 & 35 & 45 & 56 & 63 & 68 & 69 & 69 & 66 & 62 & 56 & 50 & 43 & 35 & 29 & 22 & 17 & 12 & 8 \\
\hline 0.40 & 21 & 26 & 33 & 40 & 46 & 50 & 52 & 52 & 50 & 47 & 43 & 38 & 33 & 28 & 23 & 18 & 14 & 10 & 8 \\
\hline 0.45 & 17 & 21 & 25 & 30 & 35 & 38 & 40 & 40 & 39 & 37 & 34 & 30 & 27 & 23 & 19 & 15 & 12 & 9 & 7 \\
\hline 0.50 & 15 & 17 & 19 & 23 & 26 & 29 & 31 & 31 & 31 & 29 & 27 & 25 & 22 & 19 & 16 & 13 & 11 & 9 & 7 \\
\hline \multicolumn{20}{|c|}{$K=5$} \\
\hline 0.05 & 1137 & 2001 & 2667 & 3147 & 3457 & 3613 & 3635 & 3541 & 3349 & 3075 & 2742 & 2365 & 1962 & 1555 & 1161 & 796 & 480 & 233 & 71 \\
\hline 0.10 & 295 & 504 & 668 & 786 & 864 & 903 & 909 & 886 & 838 & 770 & 688 & 595 & 494 & 395 & 296 & 206 & 128 & 67 & 26 \\
\hline 0.15 & 136 & 227 & 298 & 350 & 384 & 401 & 404 & 394 & 373 & 344 & 308 & 267 & 223 & 178 & 136 & 97 & 62 & 35 & 18 \\
\hline 0.20 & 82 & 128 & 168 & 196 & 216 & 225 & 227 & 222 & 211 & 195 & 175 & 152 & 128 & 103 & 81 & 59 & 39 & 25 & 15 \\
\hline 0.25 & 56 & 81 & 108 & 125 & 137 & 144 & 145 & 142 & 135 & 125 & 113 & 99 & 84 & 69 & 54 & 41 & 29 & 19 & 13 \\
\hline 0.30 & 43 & 55 & 73 & 87 & 95 & 100 & 101 & 99 & 94 & 88 & 79 & 70 & 60 & 50 & 40 & 31 & 23 & 16 & 12 \\
\hline 0.35 & 33 & 41 & 52 & 63 & 69 & 73 & 74 & 73 & 70 & 65 & 59 & 53 & 46 & 38 & 31 & 25 & 19 & 14 & 11 \\
\hline 0.40 & 27 & 32 & 39 & 46 & 52 & 55 & 57 & 56 & 54 & 51 & 46 & 41 & 36 & 31 & 26 & 21 & 17 & 13 & 11 \\
\hline 0.45 & 22 & 25 & 30 & 35 & 40 & 43 & 44 & 44 & 43 & 40 & 37 & 34 & 30 & 26 & 22 & 18 & 15 & 12 & 10 \\
\hline 0.50 & 19 & 21 & 24 & 27 & 31 & 34 & 35 & 36 & 35 & 33 & 31 & 28 & 25 & 22 & 19 & 16 & 14 & 12 & 10 \\
\hline \multicolumn{20}{|c|}{$K=10$} \\
\hline 0.05 & 1178 & 2026 & 2683 & 3159 & 3467 & 3622 & 3643 & 3547 & 3354 & 3081 & 2746 & 2371 & 1968 & 1560 & 1164 & 799 & 484 & 237 & 75 \\
\hline 0.10 & 329 & 528 & 685 & 799 & 874 & 911 & 916 & 892 & 844 & 776 & 693 & 599 & 498 & 398 & 300 & 209 & 131 & 70 & 30 \\
\hline 0.15 & 157 & 248 & 313 & 362 & 393 & 409 & 411 & 400 & 379 & 349 & 312 & 271 & 227 & 183 & 140 & 100 & 66 & 40 & 22 \\
\hline 0.20 & 97 & 144 & 183 & 208 & 225 & 234 & 234 & 228 & 216 & 200 & 179 & 157 & 132 & 107 & 84 & 62 & 43 & 29 & 19 \\
\hline 0.25 & 69 & 93 & 120 & 137 & 148 & 152 & 153 & 149 & 141 & 131 & 118 & 104 & 88 & 73 & 58 & 44 & 33 & 24 & 17 \\
\hline 0.30 & 53 & 66 & 83 & 97 & 105 & 108 & 108 & 106 & 100 & 94 & 84 & 75 & 64 & 54 & 44 & 35 & 27 & 21 & 17 \\
\hline 0.35 & 42 & 49 & 61 & 71 & 78 & 81 & 82 & 80 & 76 & 71 & 65 & 58 & 50 & 43 & 36 & 30 & 24 & 19 & 16 \\
\hline 0.40 & 34 & 39 & 46 & 54 & 60 & 63 & 64 & 63 & 60 & 56 & 52 & 46 & 41 & 36 & 30 & 26 & 22 & 18 & 15 \\
\hline 0.45 & 29 & 32 & 36 & 42 & 47 & 50 & 51 & 51 & 49 & 46 & 43 & 39 & 35 & 31 & 27 & 23 & 20 & 17 & 15 \\
\hline 0.50 & 25 & 27 & 30 & 33 & 37 & 40 & 42 & 42 & 41 & 39 & 37 & 34 & 30 & 27 & 24 & 21 & 19 & 17 & 15 \\
\hline
\end{tabular}

Note. $\mathrm{P}^{2}$ is the population squared multiple correlation coefficient, $\omega$ is the desired confidence interval width, and $K$ is the number of regressor variables. 
TABLE 5

Necessary Sample Size per Group for 95\% Confidence Intervals for the Population Squared Multiple Correlation Coefficient for Selected Situations When the Number of Regressors Equals 2, 5, and 10 With a Desired Degree of Assurance of .99 That the Observed Width Will Be Less Than the Desired Width

\begin{tabular}{|c|c|c|c|c|c|c|c|c|c|c|c|c|c|c|c|c|c|c|c|}
\hline \multirow[b]{2}{*}{$\omega$} & \multicolumn{19}{|c|}{$P^{2}$} \\
\hline & 0.05 & 0.10 & 0.15 & 0.20 & 0.25 & 0.30 & 0.35 & 0.40 & 0.45 & 0.50 & 0.55 & 0.60 & 0.65 & 0.70 & 0.75 & 0.80 & 0.85 & 0.90 & 0.95 \\
\hline \multicolumn{20}{|c|}{$K=2$} \\
\hline 0.05 & 1609 & 2394 & 2963 & 3343 & 3566 & 3641 & 3641 & 3608 & 3472 & 3241 & 2938 & 2583 & 2192 & 1786 & 1374 & 988 & 642 & 350 & 133 \\
\hline 0.10 & 505 & 677 & 799 & 872 & 906 & 910 & 910 & 909 & 891 & 844 & 782 & 699 & 604 & 505 & 401 & 299 & 205 & 124 & 56 \\
\hline 0.15 & 261 & 333 & 374 & 398 & 404 & 404 & 404 & 404 & 402 & 388 & 365 & 331 & 293 & 249 & 202 & 157 & 113 & 71 & 36 \\
\hline 0.20 & 166 & 199 & 218 & 226 & 227 & 227 & 227 & 227 & 227 & 224 & 214 & 198 & 176 & 155 & 128 & 102 & 74 & 51 & 27 \\
\hline 0.25 & 116 & 134 & 143 & 145 & 145 & 145 & 145 & 145 & 145 & 144 & 141 & 133 & 121 & 106 & 90 & 74 & 56 & 38 & 23 \\
\hline 0.30 & 88 & 96 & 100 & 100 & 100 & 100 & 100 & 100 & 100 & 100 & 99 & 96 & 89 & 80 & 69 & 57 & 44 & 32 & 20 \\
\hline 0.35 & 69 & 72 & 73 & 73 & 73 & 73 & 73 & 73 & 73 & 73 & 73 & 72 & 68 & 62 & 53 & 47 & 37 & 27 & 18 \\
\hline 0.40 & 55 & 56 & 56 & 56 & 56 & 56 & 56 & 56 & 56 & 56 & 56 & 56 & 54 & 50 & 45 & 39 & 31 & 24 & 16 \\
\hline 0.45 & 44 & 44 & 44 & 44 & 44 & 44 & 44 & 44 & 44 & 44 & 44 & 44 & 43 & 41 & 38 & 33 & 27 & 21 & 14 \\
\hline 0.50 & 36 & 36 & 35 & 35 & 35 & 35 & 35 & 35 & 35 & 35 & 35 & 35 & 35 & 34 & 32 & 28 & 24 & 19 & 13 \\
\hline \multicolumn{20}{|c|}{$K=5$} \\
\hline 0.05 & 1635 & 2404 & 2971 & 3353 & 3575 & 3645 & 3646 & 3613 & 3475 & 3244 & 2938 & 2588 & 2192 & 1790 & 1374 & 994 & 645 & 353 & 137 \\
\hline 0.10 & 520 & 690 & 805 & 876 & 911 & 914 & 914 & 914 & 895 & 849 & 785 & 702 & 608 & 505 & 402 & 300 & 209 & 128 & 60 \\
\hline 0.15 & 276 & 340 & 380 & 403 & 408 & 408 & 408 & 408 & 406 & 392 & 368 & 335 & 297 & 252 & 206 & 160 & 117 & 76 & 41 \\
\hline 0.20 & 177 & 207 & 224 & 231 & 231 & 231 & 231 & 231 & 231 & 228 & 217 & 201 & 180 & 156 & 131 & 103 & 80 & 56 & 33 \\
\hline 0.25 & 126 & 140 & 148 & 149 & 149 & 149 & 149 & 149 & 149 & 149 & 145 & 135 & 123 & 109 & 93 & 76 & 61 & 44 & 28 \\
\hline 0.30 & 97 & 103 & 105 & 105 & 105 & 105 & 105 & 105 & 105 & 105 & 104 & 99 & 91 & 82 & 72 & 59 & 51 & 37 & 25 \\
\hline 0.35 & 75 & 78 & 78 & 78 & 78 & 78 & 78 & 78 & 78 & 78 & 78 & 76 & 71 & 65 & 57 & 48 & 43 & 33 & 23 \\
\hline 0.40 & 60 & 61 & 61 & 61 & 61 & 61 & 61 & 61 & 61 & 61 & 61 & 60 & 57 & 53 & 47 & 41 & 38 & 30 & 22 \\
\hline 0.45 & 49 & 49 & 49 & 49 & 49 & 49 & 49 & 49 & 49 & 49 & 49 & 48 & 47 & 45 & 41 & 36 & 34 & 28 & 21 \\
\hline 0.50 & 43 & 40 & 40 & 40 & 40 & 40 & 40 & 40 & 40 & 40 & 40 & 40 & 40 & 38 & 35 & 31 & 31 & 26 & 20 \\
\hline \multicolumn{20}{|c|}{$K=10$} \\
\hline 0.05 & 1651 & 2425 & 2981 & 3362 & 3579 & 3653 & 3653 & 3620 & 3480 & 3251 & 2947 & 2592 & 2199 & 1789 & 1378 & 994 & 643 & 351 & 135 \\
\hline 0.10 & 542 & 705 & 817 & 887 & 919 & 922 & 922 & 921 & 900 & 855 & 789 & 707 & 613 & 509 & 405 & 304 & 206 & 125 & 58 \\
\hline 0.15 & 296 & 354 & 391 & 411 & 416 & 416 & 416 & 416 & 413 & 399 & 373 & 339 & 301 & 255 & 211 & 163 & 113 & 74 & 38 \\
\hline 0.20 & 192 & 219 & 233 & 239 & 239 & 239 & 239 & 239 & 239 & 234 & 222 & 206 & 183 & 161 & 134 & 107 & 77 & 52 & 29 \\
\hline 0.25 & 139 & 151 & 156 & 157 & 157 & 157 & 157 & 157 & 157 & 156 & 151 & 142 & 130 & 113 & 98 & 80 & 58 & 41 & 25 \\
\hline 0.30 & 107 & 111 & 112 & 112 & 112 & 112 & 112 & 112 & 112 & 112 & 110 & 105 & 96 & 87 & 75 & 63 & 46 & 34 & 22 \\
\hline 0.35 & 85 & 86 & 86 & 86 & 86 & 86 & 86 & 86 & 86 & 86 & 85 & 82 & 77 & 70 & 62 & 53 & 39 & 29 & 19 \\
\hline 0.40 & 68 & 68 & 68 & 68 & 68 & 68 & 68 & 68 & 68 & 68 & 68 & 67 & 63 & 58 & 52 & 46 & 34 & 26 & 18 \\
\hline 0.45 & 56 & 56 & 56 & 56 & 56 & 56 & 56 & 56 & 56 & 56 & 56 & 56 & 54 & 50 & 46 & 40 & 30 & 23 & 16 \\
\hline 0.50 & 48 & 48 & 48 & 48 & 48 & 48 & 48 & 48 & 48 & 48 & 48 & 48 & 47 & 44 & 41 & 36 & 27 & 22 & 16 \\
\hline
\end{tabular}

Note. $\mathrm{P}^{2}$ is the population squared multiple correlation coefficient, $\omega$ is the desired confidence interval width, and $K$ is the number of regressor variables. 


\section{EXAMPLE OF PLANNING SAMPLE SIZE FOR P2 FROM THE AIPE APPROACH USING MBESS}

As a way of solidifying the methods discussed, an illustrative example based on the work of Bunce and West (1995) is provided, which was previously used as an example in the context of power analysis by Murphy and Myors (2004, pp. 93-97). One question of interest to Bunce and West was linking innovation among health service workers to previous innovation, personality factors (intrinsic job motivation, propensity to innovate, and rule independence), and group climate factors (participation in decision making, shared vision, support for innovation, \& task orientation). Bunce and West found that in their analysis of 77 participants, nearly $40 \%$ of the variance in innovation among health service workers could be accounted for by the eight regressors of interest $\left(R^{2}=\right.$ $.39 ; F(8,68)=5.38, p<.001)$. A confidence interval for $\mathrm{P}^{2}$ based on the summary values provided by Bunce $\&$ West reveals that, although they obtained statistically significant results, $\mathrm{P}^{2}$ was not estimated with much precision, as the $95 \%$ confidence interval for $\mathrm{P}^{2}$ ranges from .140 to .503 (a width of .363). Confidence intervals for $\mathrm{P}^{2}$ when regressors are random can be obtained with the ci.R2 () function from the MBESS (Kelley, 2007a, 2007b, 2008; Kelley et al., 2008) R package (R Development Core Team, 2008). ${ }^{7}$ Both $\mathrm{R}$ and MBESS are Open Source and freely available. A call to the ci.R2() function for the example would be of the form

$$
\text { ci.R2(R2 } \left.=R^{2}, \mathrm{~N}=N, \mathrm{~K}=K \text {, conf.level }=1-\alpha\right) .
$$

The ci.R2 () would be implemented as follows for the Bunce and West (1995) example:

$$
\text { ci.R2(R2 }=.39, \mathrm{~N}=77, \mathrm{~K}=8, \text { conf.level }=.95),
$$

which yields .140 and .503 for the lower and upper 95\% confidence limits, respectively.

Suppose that a researcher would like to build on the Bunce and West (1995) work with a goal of achieving a narrow $95 \%$ confidence interval for $\mathrm{P}^{2}$. Using the findings from Bunce \& West and a triangulation of other sources, suppose that the researchers find support for $\mathrm{P}^{2}$ approximating .40 . In an effort to obtain a narrow confidence interval for $\mathrm{P}^{2}$ in their study, say a confidence interval

${ }^{7} \mathrm{R}$ and MBESS are both available from the Comprehensive $\mathrm{R}$ Archival Network (CRAN) at www.cran.r-project.com and http://cran.r-project.org/src/contrib/ Descriptions/MBESS.html, respectively. On Macintosh and Windows systems, MBESS can be installed from within $\mathrm{R}$ directly using the Package Installation feature, which connects to the CRAN where the software is housed. Source code for Macintosh, Windows, Linux/Unix is available on CRAN. 
that has a width of .10 , the researchers follow the approach discussed in this article and plan sample size from the AIPE perspective. At present, the easiest way to implement the methods discussed and developed in this article is to use the MBESS R package. The function used to plan sample size from the AIPE perspective for $\mathrm{P}^{2}$ is ss.aipe.R2(). A basic call to the ss . aipe.R2() function would be of the form

ss.aipe.R2(Population.R2 $=\mathrm{P}^{2}$, width $=\omega, \mathrm{K}=K$, conf.level $=1-\alpha$ ).

The present example would thus be operationalized using the ss.aipe.R2() a function s follows:

ss.aipe.R2(Population.R2 $=.40$, width $=.10, \mathrm{~K}=8$, conf.level $=.95)$,

which yields a necessary sample size of 893 . However, as discussed, the sample size obtained in this fashion should be regarded as approximate. To determine the exact value of sample size, an a priori Monte Carlo simulation study should be used, which can be implemented with specification of the verify.ss=TRUE argument in the ss.aipe.R2() function:

ss.aipe. R2 (Population. R2=.40, width $=.10, \mathrm{~K}=8$, conf. level=.95, verify.ss=TRUE),

which reveals that the exact sample size, such that the expected width of the confidence interval for $\mathrm{P}^{2}$ is .10 units wide, is 889 . Although the approximate method yields essentially the correct value, it reveals a sample size that is larger than necessary by 4 . Thus, using a sample size of 889 in the situation described will lead to a confidence interval for $\mathrm{P}^{2}$ whose expected width is .10 (provided all necessary parameters are properly specified and assumptions met).

Although designing a study so that the expected width is sufficiently narrow is desirable, the researchers investigate the implications on sample size when an assurance parameter (i.e., $\gamma$ ) of .99 is also included, as doing so will ensure that the obtained confidence interval is no wider than .10 with no less than .99 probability. The way in which sample size can be planned in this case is by specifying assurance $=.99$ in the ss.aipe.R2() R function. The approximate method can be implemented as

ss . aipe. $R 2$ (Population. $R 2=.40$, width $=.10, \mathrm{~K}=8$, conf.level=.95, assurance $=.99$ ),

which yields a necessary sample size of 919. As before, incorporating the verify.ss=TRUE argument implements the a priori Monte Carlo simulation 
study so that the exact sample size can be found. The ss . aipe.R2() R function would be used as follows:

ss.aipe. $\mathrm{R} 2$ (Population. $\mathrm{R} 2=.40$, width $=.10, \mathrm{~K}=8$, conf. level=.95, assurance=.99, verify.ss=TRUE),

which returns a necessary sample size of 918 . The exact approach shows that the approximate method overestimates the necessary sample size by 1 . Thus, using a sample size of 918 in the situation described ensures with no less than .99 assurance that the observed confidence interval will not be larger than .10 (provided all necessary parameters are properly specified and assumptions met). It is interesting to note that had a desired confidence interval width of .20 been specified instead of .10 in this situation, necessary sample size would have been 226 and 236, respectively, for the expected confidence interval width and incorporating an assurance of .99 for the exact approach. Thus, by doubling the width of the confidence interval the necessary sample sizes are approximately quartered, a common relationship in sample size planning for narrow confidence intervals.

Sample size planning procedures almost always require one or more population parameters to be specified. However, the population parameter(s) are generally unknown, and at least with the AIPE approach to sample size planning, their value(s) are what is of interest. Thus, in all likelihood one will not know $\mathrm{P}^{2}$ exactly, which will necessarily make the whole sample size planning endeavor approximate. $^{8}$

\section{DISCUSSION}

Multiple regression is one of the most commonly used statistical procedures in the BEMS sciences. The value of $\mathrm{P}^{2}$ is often of considerable interest to researchers using multiple regression, as knowing the proportion of the variance in $Y$ that is accounted for by the $K$ regressor variables provides information on the adequacy of model fit. Although this relationship is often evaluated with a null hypothesis significance test in order to determine if the null hypothesis that $\mathrm{P}^{2}=0$ (or some other specified value) can be rejected, the benefits that confidence intervals can provide above and beyond null hypothesis significance tests have been clearly delineated in the methodological literature. As such, the

\footnotetext{
${ }^{8}$ A sensitivity analysis is often beneficial, where a variety of values for parameters are used to assess the effect of misspecifying the parameters on the desired outcome (in this case the confidence interval width). The function ss.aipe.R2.sensitivity() from the MBESS R package can be used to assess the effects of misspecified parameter values in a variety of situations.
} 
use of null hypothesis significance testing as a be-all and end-all way to draw inferential conclusions is beginning to wane to a degree in favor of confidence intervals. Wide confidence intervals are not generally desirable, which is where the AIPE approach can provide beneficial methods of planning sample size.

This article developed methods of sample size planning for the squared multiple correlation coefficient from the AIPE perspective. The first method developed allows sample size to be planned so that the expected confidence interval width for $\mathrm{P}^{2}$ will be sufficiently narrow. Choosing sample size in order for the expected value of the confidence interval for $\mathrm{P}^{2}$ to be sufficiently narrow does not imply that any particular confidence interval will be sufficiently narrow. Therefore, a modified sample size procedure was developed with the goal of obtaining a sufficiently narrow confidence interval with some desired degree of assurance. A procedure for fine-tuning the sample size from the originally proposed procedure to obtain the exact value with an a priori Monte Carlo simulation was also discussed. The a priori Monte Carlo simulation study is used to evaluate the effect of a particular sample size on various aspects of the confidence interval width. The simulation can be performed using a systematic search of sample sizes until the minimum value of sample size is found that satisfies the stated research goals.

Sample size planning for the squared multiple correlation coefficient has largely been from a power analytic perspective. However, because researchers are often interested in the value of $\mathrm{P}^{2}$ itself, calculation of sample size given the goals of AIPE is at times more appropriate than the power analytic approach. The AIPE approach to sample size planning and the power analytic approach each have their place. However, it should be clear that the goals of AIPE and the power analytic approach are fundamentally different, which has the effect of necessary sample sizes potentially being very different under different scenarios. Depending on the question of interest and the goals of the study, one approach may be more appropriate. Of course, when there is a desire to have narrow confidence intervals and an estimate that leads to statistical significance, both AIPE and the power analytic approach can be used. In such a situation it is recommended that the larger of the two sample sizes be used so that issues of both power and accuracy can be addressed.

Recall that the procedures for confidence interval formation for $\mathrm{P}^{2}$ assumes multivariate normality. Because the sample size planning procedures are based on the confidence interval formation methods, they also assume multivariate normality. What is not clear at present is the effectiveness of the sample size planning procedures when data are sampled from a population where multivariate normality does not hold. Among other things, Kromrey and Hess (2001) evaluated the confidence interval formation procedure for $\mathrm{P}^{2}$ under a variety of conditions, one of which was when multivariate normality did not hold. Under nonnormality, the results of a Monte Carlo simulation study conducted by 
Kromrey and Hess were mixed. In general, if the confidence interval procedure itself does not work well in cases of nonnormality, it is unlikely that the proposed sample size planning procedures discussed here will work well in cases of nonnormality. Evaluation of the robustness of the sample size planning procedure when data are not multivariate normal is a worthwhile future research topic, as distributions in the BEMS sciences often deviate from normality (e.g., Micceri, 1989).

Whenever an estimate is of interest, so too should the corresponding confidence interval for the population quantity. Holding everything else constant, the wider a confidence interval the greater the uncertainty of the value of the population parameter of interest. However, holding everything else constant, the narrower a confidence interval the smaller the range of plausible values for the parameter of interest. Determining the parameter value is often one of the major goals of a research study. Therefore, it is hoped that during the design phase of research that will use multiple regression, the AIPE approach to sample size planning be seriously considered in an effort to obtain a confidence interval for $\mathrm{P}^{2}$ that is sufficiently narrow.

\section{REFERENCES}

Algina, J., \& Olejnik, S. (2000). Determining sample size for accurate estimation of the squared multiple correlation coefficient. Multivariate Behavioral Research, 35, 119-136.

Bunce, D., \& West, M. A. (1995). Self perceptions and perceptions of group climate as predictors of individual innovation at work. Applied Psychology: An International Review, 44, 199-215.

Cohen, J. (1988). Statistical power analysis for the behavioral sciences (2nd ed.). Hillsdale, NJ: Erlbaum.

Cohen, J. (1994). The earth is round ( $p<.05)$. American Psychologist, 49, 997-1003.

Darlington, R. B. (1990). Regression and linear models. New York: McGraw-Hill.

Ding, C. G. (1996). On the computation of the distribution of the square of the sample multiple correlation coefficient. Computational Statistics \& Data Analysis, 22, 345-350.

Dunlap, W. P., Xin, X., \& Myers, L. (2004). Computing aspects of power for multiple regression. Behavior Research Methods, Instruments, \& Computers, 36, 695-701.

Gatsonis, C., \& Sampson, A. R. (1989). Multiple correlation: Exact power and sample size calculations. Psychological Bulletin, 106, 516-524.

Green, S. B. (1991). How many subjects does it take to do a regression analysis? Multivariate Behavioral Research, 26, 499-510.

Hedges, L. \& Olkin, I. (1985). Statistical methods for meta-analysis. Orlando, FL: Academic.

Johnson, N. L., Kotz, S., \& Balakrishnan, N. (1995). Continuous univariate distributions (2nd ed., Vol. 2). New York: Wiley.

Joseph, L., Berger, R. du, \& Bélisle, P. (1995). Sample size calculations for binomial proportions via highest posterior density intervals. The Statistician, 44, 143-154.

Kelley, K. (2007a). Confidence intervals for standardized effect sizes: Theory, application, and implementation. Journal of Statistical Software, 20(8), 1-24.

Kelley, K. (2007b). Methods for the behavioral, educational, and social sciences: An R package. Behavior Research Methods, 39, 979-984. 
Kelley, K. (2007c). Sample size planning for the coefficient of variation from the accuracy in parameter estimation approach. Behavior Research Methods, 39, 755-766.

Kelley, K. (2008). MBESS version 2.0 [Computer software and manual]. Retrieved from http://www. cran.r-project.org/

Kelley, K., Lai, K., \& Wu, P.-J. (2008). Using R for data analysis: A best practice for research. In J. Osbourne (Ed.), Best practices in quantitative methods (pp. 535-572). Newbury Park, CA: Sage.

Kelley, K., \& Maxwell, S. E. (2003). Sample size for multiple regression: Obtaining regression coefficients that are accurate, not simply significant. Psychological Methods, 8, 305-321.

Kelley, K., \& Maxwell, S. E. (2008). Sample size planning for multiple regression: Power and accuracy for omnibus and targeted effects. In J. Brannon, P. Alasuutari, \& L. Bickman (Eds.), Sage handbook of social research methods (pp. 166-192). Newbury Park, CA: Sage.

Kelley, K., Maxwell, S. E., \& Rausch, J. R. (2003). Obtaining power or obtaining precision: Delineating methods of sample size planning. Evaluation and the Health Professions, 26, 258287.

Kelley, K., \& Rausch, J. R. (2006). Sample size planning for the standardized mean difference: Accuracy in parameter estimation via narrow confidence intervals. Psychological Methods, 11, 363-385.

Kromrey, J. D., \& Hess, M. (2001, April). Confidence bands for $r^{2}$ : An empirical comparison of accuracy and precision with non-normal populations. Paper presented at the annual meeting of the American Educational Research Association, Seattle, WA.

Lee, Y. S. (1971). Tables of the upper percentage points of the multiple correlation. Biometrika, 59, 175-189.

Maxwell, S. E., Kelley, K., \& Rausch, J. R. (2008). Sample size planning for statistical power and accuracy in parameter estimation. Annual Review of Psychology, 59, 537-563.

Mendoza, J. L., \& Stafford, K. L. (2001). Confidence intervals, power calculations, and sample size estimation for the squared multiple correlation coefficient under the fixed and random regression models: A computer program and useful standard tables. Educational and Psychological Measurement, 61, 650-667.

Micceri, T. (1989). The unicorn, the normal curve, and other improbable creatures. Psychological Bulletin, 105, 156-166.

M'Lan, C. E., Joseph, L., \& Wolfson, D. B. (2006). Bayesian sample size determination for casecontrol studies. Journal of the American Statistical Association, 101, 760-772.

Murphy, K. R., \& Myors, B. (2004). Statistical power analysis: A simple and general model for traditional and modern hypothesis tests (2nd ed.). Mahwah, NJ: Erlbaum.

Muthén, L., \& Muthén, B. (2002). How to use a Monte Carlo study to decide on sample size and determine power. Structural Equation Modeling, 9, 599-620.

Neyman, J. (1937). Outline of a theory of statistical estimation based on the classical theory of probability. Philosophical Transaction of the Royal Society of London. Series A, Mathematical and Physical Sciences, 236, 333-380.

O'Brien, R., \& Muller, K. (1993). A unified approach to statistical power for $t$-tests to multivariate models. In L. K. Edwards (Ed.), Applied analysis of variance in behavioral sciences (pp. 297344). New York: Marcel Dekker.

R Development Core Team. (2008). R: A language and environment for statistical computing. Vienna. Rencher, A. C. (2000). Linear models in statistics. New York: Wiley.

Sampson, A. R. (1974). A tale of two regressions. Journal of the American Statistical Association, 69, 682-689.

Satorra, A., \& Saris, W. E. (1985). Power of the likelihood ratio test in covariance structure analysis. Psychometrika, 50, 83-90. 
Smithson, M. (2001). Correct confidence intervals for various regression effect sizes and parameters: The importance of noncentral distributions in computing intervals. Educational and Psychological Measurement, 61, 605-632.

Steiger, J. H. (2004). Beyond the $F$ test: Effect size confidence intervals and tests of close fit in the analysis of variance and contrast analysis. Psychological Methods, 9, 164-182.

Steiger, J. H., \& Fouladi, R. T. (1992). R2: A computer program for interval estimation, power calculation, and hypothesis testing for the squared multiple correlation. Behavior Research Methods, Instruments, and Computers, 4, 581-582.

Stuart, A., Ord, J. K., \& Arnold, S. (1999). Kendall's advanced theory of statistics: Classical inference and the linear model (6th ed., Vol. 2A). New York: Oxford University Press.

Task Force on Reporting of Research Methods in AERA Publications. (2006). Standards for reporting on empirical social science research in AERA publications, American educational. Washington, DC: American Educational Research Association.

Thompson, B. (2002). What future quantitative social science research could look like: Confidence intervals for effect sizes. Educational Researcher, 31, 25-32.

Wang, F., \& Gelfand, A. E. (2002). A simulation-based approach to Bayesian sample size determination for performance under a given model and for separating models. Statistical Science, 193-208.

Wilkinson, L., \& the American Psychological Association Task Force on Statistical Inference. (1999). Statistical methods in psychology: Guidelines and explanations. American Psychologist, $54,594-604$. 